\title{
THE VALUATION OF LIFE CONTINGENCIES: A SYMMETRICAL TRIANGULAR FUZZY APPROXIMATION
}

Jorge de Andrés-Sánchez ${ }^{\mathrm{a}, 1}$

a Department of Business Administration. Faculty of Economics and Business Studies. Rovira i Virgili University, Av. de la Universitat 1, 43204 Reus, Spain. E-mail: jorge.deandres@urv.cat

${ }^{1}$ Corresponding author

Laura González-Vila Puchades ${ }^{b}$

b Department of Mathematics for Economics, Finance and Actuarial Science. Faculty of Economics and Business. University of Barcelona, Av. Diagonal 690, 08034 Barcelona, Spain. E-mail: Igonzalezv@ub.edu

\begin{abstract}
This paper extends the framework for the valuation of life insurance policies and annuities by Andrés-Sánchez and González-Vila $(2012,2014)$ in two ways. First, we allow various uncertain magnitudes to be estimated by means of fuzzy numbers. This applies not only to interest rates but also to the amounts to be paid out by the insurance company. Second, the use of symmetrical triangular fuzzy numbers allows us to obtain expressions for the pricing of life contingencies and their variability that are closely linked to standard financial and actuarial mathematics. Moreover, they are relatively straightforward to compute and understand from a standard actuarial point of view.
\end{abstract}

JEL classification codes: G22, C63

KEYWORDS: Life contingency pricing, fuzzy numbers, fuzzy random variables, fuzzy financial mathematics.

\section{INTRODUCTION}

Stochastic techniques are, beyond doubt, at the core of actuarial mathematics. However, in insurance decision-making problems, as well as in other areas related to economics and finance, much of the information is imprecise and vague, or relies heavily on subjective judgements and, so, it is not clearly measurable. For such information, the use of fuzzy set theory (FST) can represent a suitable alternative and/or a supplementary way to that of pure statistical methods as has been shown in De Witt (1982), Lemaire (1990), Ostaszewski (1993), Cummins and Derrig (1997), Andrés-Sánchez and Terceño (2003) and Shapiro (2004). 
In the field of the financial pricing of insurance, FST has been used to model discount rates. Cummins and Derrig (1997), Derrig and Ostaszewski (1997) and Andrés-Sánchez (2014) do so in a non-life context, while Lemaire (1990), Ostaszewski (1993) and Betzuen et al. (1997) model discount rates for life insurance contingencies valuation. In these papers probabilities, however, are reduced to predefined frequencies and so, the financial pricing of insurance contracts is solved by applying the fuzzy financial mathematics developed by Buckley (1987). Anyway, when applying these methods, probabilistic information is lost because random magnitudes are reduced to their mathematical expectation.

Shapiro (2009) exposes the concept of fuzzy random variables (FRVs) with Actuarial Science in view. Similarly, Huang et al. (2009) develop an individual risk model in which the cost of accidents is estimated using fuzzy numbers (FNs), while the number of claims follows a Poisson process. In the field of life insurance, Andrés-Sánchez and González-Vila (2012, 2014) develop a methodology in which discount rates are fuzzy whereas the mortality is strictly random. In these papers, the stochastic modelling of life contingencies with deterministic discount rates and monetary amounts (see Wolthuis and Van Hoek (1986) for a complete description) is extended to cases in which the discount rates are fuzzy and, so, the outcomes (the present value of insured life contingencies) are fuzzy sets. All these developments also rely on the concept of FRVs.

This paper extends the previous findings of Andrés-Sánchez and González-Vila (2012, 2014) in two ways. First, we also allow the insured amounts to be paid out by the insurance company to be uncertain and to be quantified with FNs. Note, that, in fact, these amounts may be linked to economic indexes, such as the consumer price index, a wage growth rate, etc. Likewise, if we are evaluating the underwriter's overall outcome of a policy, future maintenance costs, general and settlement expenses, etc. may not be known exactly a priori.

Second, we suppose that the amounts to be paid and the interest rates are fitted with symmetrical triangular fuzzy numbers (STFNs). Indeed, the use of these FNs is very common in the fuzzy literature. In a strictly actuarial context, we find Andrés-Sánchez and Terceño (2003), Shapiro (2013) and Heberle and Thomas (2014). This approach allows us to deduce several operational results that are relatively easy to implement and understand with standard actuarial skills, as they rely on conventional statistical and financial concepts.

We structure the rest of our paper as follows. In section 2 we describe the concepts and instruments of FST used in our developments: FNs and FRVs. We then develop a STFN approximation for the present value derived from STFN cash-flows and discount rates with a straightforward financial interpretation. In sections 4 and 5 we introduce the use of FRV with STFN outcomes to price life contingencies. We conclude our paper with a summary of the main conclusions and possible extensions. 


\section{FUZZY NUMBERS AND FUZZY RANDOM VARIABLES}

\subsection{FUZZY NUMBERS AND FUZZY ARITHMETIC}

In this section we describe the basic concepts of FST and FNs and so present the basic notation used throughout this paper. The basic concept on which FST is based is the fuzzy set. A fuzzy set $\widetilde{A}$ can be defined as $\widetilde{A}=\left\{\left(x, \mu_{\tilde{A}}(x)\right) \mid x \in X\right\}$, where $\mu_{\tilde{A}}$ is known as the membership function and is a mapping from the referential set $X$ to the interval $[0,1]$, i.e. $\mu_{\tilde{A}}: X \rightarrow[0,1]$. Therefore, 0 indicates non-membership of the fuzzy subset $\widetilde{A}$ and 1 indicates absolute membership. Alternatively, a fuzzy set can be represented by its $\alpha$-level sets or $\alpha$ cuts. An $\alpha$-cut is a crisp set $A_{\alpha}$, where $A_{\alpha}=\left\{x \in X \mid \mu_{\tilde{A}}(x) \geq \alpha\right\}, \forall \alpha \in(0,1]$, with the convention that $A_{\alpha=0}$ is the closure of the support of $\widetilde{A}$, i.e. all $x \in X$ that $\mu_{\widetilde{A}}(x) \geq 0$.

A fuzzy number is a fuzzy subset $\widetilde{A}$ defined over the set of real numbers and it is a fundamental concept of FST for representing uncertain quantities. It is normal, i.e. $\max _{x \in X} \mu_{\tilde{A}}(x)=1$, and convex, that is, its $\alpha$-cuts are closed and bounded intervals. So, they are $A_{\alpha}=[\underline{A}(\alpha), \bar{A}(\alpha)], 0 \leq \alpha \leq 1$. In this paper we use symmetrical triangular fuzzy numbers, which we denote as $\widetilde{A}=\left(A, r_{A}\right)$. The value $A$ is the core of the FN, i.e. $\mu_{\tilde{A}}(A)=$ 1 whereas $r_{A}$ is the spread. Thus, the membership function and its corresponding $\alpha$-cuts are:

$$
\begin{gathered}
\mu_{\tilde{A}}(x)=\max \left\{0, \frac{|x-A|}{r_{A}}\right\} \\
A_{\alpha}=[\underline{A}(\alpha), \bar{A}(\alpha)]=\left[A-r_{A}(1-\alpha), A+r_{A}(1-\alpha)\right]
\end{gathered}
$$

The expected value of the FN $\widetilde{A}, E V(\widetilde{A} ; \lambda)$, is a representative real value of this FN that was developed in Campos and González (1989). This concept allows us to introduce the risk aversion of the decision maker with a coefficient $0 \leq \lambda \leq 1$ in such a way that:

$$
E V(\widetilde{A} ; \lambda)=(1-\lambda) \int_{0}^{1} \underline{A}(\alpha) d \alpha+\lambda \int_{0}^{1} \bar{A}(\alpha) d \alpha
$$

So, for a STFN $\widetilde{A}=\left(A, r_{A}\right)$ it is straightforward to check that:

$$
E V(\widetilde{A} ; \lambda)=A+r_{A}\left(\lambda-\frac{1}{2}\right)
$$

Let $f(\cdot)$ be a continuous real valued function of $n$-real variables $x_{j}, j=1,2, \ldots, n$, and let $\tilde{A}_{1}, \tilde{A}_{2}, \ldots, \tilde{A}_{n} n$ FNs. Then Zadeh's extension principle in Zadeh (1965) allows us to define a FN $\tilde{B}$ induced by the FNs $\tilde{A}_{1}, \tilde{A}_{2}, \ldots, \tilde{A}_{n}$ through $f(\cdot)$ as $\tilde{B}=f\left(\tilde{A}_{1}, \tilde{A}_{2}, \ldots, \tilde{A}_{n}\right)$.

Although it is usually impossible to obtain the membership function of $\widetilde{B}$, it is often possible to obtain a closed expression for its $\alpha$-cuts, $B_{\alpha}$. If $f(\cdot)$ is increasing with respect to the first $m$ variables, $m \leq n$, and decreases in the last $n-m$ variables, Buckley and $\mathrm{Qu}$ (1990) demonstrate that:

$$
B_{\alpha}=[\underline{B}(\alpha), \bar{B}(\alpha)]=\left[f\left(\underline{A}_{1}(\alpha), \underline{A}_{2}(\alpha), \ldots, \underline{A}_{m}(\alpha), \bar{A}_{m+1}(\alpha), \bar{A}_{m+2}(\alpha), \ldots \bar{A}_{n}(\alpha)\right),\right.
$$




$$
\left.f\left(\bar{A}_{1}(\alpha), \bar{A}_{2}(\alpha), \ldots \bar{A}_{m}(\alpha), \underline{A}_{m+1}(\alpha), \underline{A}_{m+2}(\alpha), \ldots, \underline{A}_{n}(\alpha)\right)\right]
$$

When $f(\cdot)$ is simply a linear combination of its variables $\sum_{j=1}^{n} k_{j} x_{j}, k_{j} \in \mathfrak{R}, j=1,2, \ldots, n$, the result of evaluating this function with $\tilde{A}_{j}=\left(A_{j}, r_{A_{j}}\right), j=1,2, \ldots, n$, is a $\operatorname{STFN} \tilde{B}=\left(B, r_{B}\right)$, where:

$$
B=\sum_{j=1}^{n} k_{j} A_{j}, r_{B}=\sum_{j=1}^{n}\left|k_{j}\right| r_{A_{j}}
$$

However, the result of evaluating non-linear functions with STFNs is not a STFN. In any case, if $f(\cdot)$ is a real-valued function increasing (decreasing) with respect to the first (last) $m(n-m)$ variables, it admits a STFN approximation that is based on the approximation to non-linear operations with L-R FNs developed in Dubois and Prade $(1980,1993)$. It is built up from the first-order Taylor polynomial expansion from the 1-cut to any $\alpha$-cut. So, let us approximate $\underline{B}(\alpha)$ in (3) from $B(1)$ using the Taylor expansion to the first degree with $\underline{B}(\alpha) \approx \underline{B}(1)+\frac{d \underline{B}(1)}{d \alpha}(\alpha-1)$. If we name the vector comprising the centers of $\tilde{A}_{j}$, $j=1,2, \ldots, n, A_{C}=\left(A_{1}, A_{2}, \ldots, A_{n}\right)$, this Taylor expansion is equivalent to:

$$
\begin{gathered}
\underline{B}(\alpha)=f\left(\underline{A}_{1}(\alpha), \underline{A}_{2}(\alpha), \ldots, \underline{A}_{m}(\alpha), \bar{A}_{m+1}(\alpha), \bar{A}_{m+2}(\alpha), \ldots \bar{A}_{n}(\alpha)\right) \approx \\
\approx f\left(A_{C}\right)-\sum_{j=1}^{m} \frac{\partial f\left(A_{C}\right)}{\partial x_{j}} r_{A_{j}}(1-\alpha)+\sum_{j=m+1}^{n} \frac{\partial f\left(A_{C}\right)}{\partial x_{j}} r_{A_{j}}(1-\alpha) \\
=f\left(A_{C}\right)-\sum_{j=1}^{n}\left|\frac{\partial f\left(A_{C}\right)}{\partial x_{j}}\right| r_{A_{j}}(1-\alpha)
\end{gathered}
$$

Analogously, for $\bar{B}(\alpha)$ we find:

$$
\begin{gathered}
\bar{B}(\alpha)=f\left(\bar{A}_{m+1}(\alpha), \bar{A}_{m+2}(\alpha), \ldots \bar{A}_{n}(\alpha), \underline{A}_{1}(\alpha), \underline{A}_{2}(\alpha), \ldots, \underline{A}_{m}(\alpha)\right) \approx \\
\approx f\left(A_{C}\right)+\sum_{j=1}^{n}\left|\frac{\partial f\left(A_{C}\right)}{\partial x_{j}}\right| r_{A_{j}}(1-\alpha)
\end{gathered}
$$

So, $\tilde{B} \approx\left(B, r_{B}\right)$ where:

$$
\begin{aligned}
B & =f\left(A_{C}\right) \\
r_{B} & =\sum_{j=1}^{n}\left|\frac{\partial f\left(A_{C}\right)}{\partial x_{j}}\right| r_{A_{j}}
\end{aligned}
$$

In an actuarial context, STFNs and this STFN approximation to any arithmetic operation are used in Andrés-Sánchez and Terceño (2003) for the pricing of life and non-life insurance, whereas Heberle and Thomas (2014) and Andrés-Sánchez and Terceño (2003) adapt chain ladder and London chain ladder reserving methods, respectively, to the use of development factors estimated by means of STFNs. 


\subsection{FUZZY RANDOM VARIABLES}

In many real situations, uncertainty is caused by a variety of factors: randomness, hazard, vagueness, inaccuracy, imprecision, etc. Stochastic variability can be described by the use of probability theory, while other types of uncertainty, such as vagueness and imprecision, can be captured with the use of fuzzy sets (Viertl and Hareter (2004)). The concept of FRVs combines both random and fuzzy uncertainty.

Roughly speaking, a FRV can be defined as a random variable (RV) whose outcomes are not real numbers (or vectors) but FNs (or fuzzy sets defined on $\mathfrak{R}^{n}$ ). It was initially proposed by Kwakernaak $(1978,1979)$, who extended the concept of RVs on $\Re$ to the case where the realizations are FNs. Kruse and Meyer (1987) subsequently enhanced Kwakernaak's concepts. Likewise, Puri and Ralescu (1986) conceptualized FRVs as a fuzzification of a random set. However, when outcomes are mapped to the real line both definitions coincide, as demonstrated in Krätchmer (2001). In an insurance context, Shapiro (2009) presented a general reflection on the potential uses of FRVs in Actuarial Science. In more specific problems, Huang et al. (2009) developed a non-life risk model in which the number of claims follows a Poisson distribution, while their monetary values are triangular FNs. Later, Shapiro (2013) models future lifetime as a FRV. Finally, AndrésSánchez and González-Vila $(2012,2014)$ apply FRVs to price life contingencies under the hypothesis of fuzzy interest rate and random behavior of mortality.

To define a discrete FRV, we consider a probability space defined by $(\Omega, \mathcal{A}, P)$ where $\Omega$ is a discrete space of elementary events $\left\{\omega_{j}\right\}, j=1,2, \ldots, n, \mathcal{A}$ is the $\sigma$-algebra of subsets of $\Omega$ and $P$ is a probability measure on $\Omega$. Additionally, we consider the Borel measurable space $(\Re, \mathcal{B})$ and we name the set of all FNs defined on $\Re$ as $F(\Re)$. The mapping $\widetilde{X}: \Omega \rightarrow F(\Re)$, where for $\forall \omega_{j} \in \Omega$ the fuzzy outcome is $\tilde{X}_{j}$ with $\alpha$-cuts $X_{j_{\alpha}}=\left[X_{j}(\alpha), \overline{X_{j}}(\alpha)\right]$, is called a fuzzy random variable $\widetilde{\boldsymbol{X}}$ if:

$$
\forall B \in \mathcal{B}, \forall \alpha \in[0,1],\left\{\omega_{j} \in \Omega \mid X_{j_{\alpha}} \cap B \neq \varnothing\right\} \in \mathcal{A}
$$

Wang and Zhang (1992) demonstrate that any FRV $\widetilde{\boldsymbol{X}}$ defines, $\forall \alpha \in[0,1]$, an infima RV $\underline{\boldsymbol{X}}(\alpha)$ and a suprema $\operatorname{RV} \overline{\boldsymbol{X}}(\alpha)$, whose realizations are, respectively, $\left\{X_{j}(\alpha)\right\}_{j=1,2, \ldots, n}$ and $\left\{\overline{X_{j}}(\alpha)\right\}_{j=1,2, \ldots, n}$. These RVs allow us to bound the distribution function of the FRV $F_{\widetilde{X}}(x)=P(\widetilde{X} \leq x)$.

Concretely, if we symbolize as $F_{\underline{X}(\alpha)}(x)$ and $F_{\overline{\boldsymbol{X}}(\alpha)}(x), \forall \alpha \in[0,1]$, the distribution functions of the $\operatorname{RVs} \underline{\boldsymbol{X}}(\alpha)$ and $\overline{\boldsymbol{X}}(\alpha)$ obtained from $\widetilde{\boldsymbol{X}}$, we define the couple of the distribution functions of the RVs infima and suprema for that membership level, $F_{\widetilde{X}}(x)_{\alpha}=$ $\left\{{\underline{F_{\widetilde{X}}(x)_{\alpha}}}, \overline{F_{\widetilde{X}}(x)}{ }_{\alpha}\right\}$, as:

$$
\begin{aligned}
& \underline{F_{\widetilde{\boldsymbol{X}}}(x)_{\alpha}}=P(\overline{\boldsymbol{X}}(\alpha) \leq x)=F_{\overline{\boldsymbol{X}}(\alpha)}(x) \\
& {\overline{F_{\widetilde{\boldsymbol{X}}}(x)}}_{\alpha}=P(\underline{\boldsymbol{X}}(\alpha) \leq x)=F_{\underline{\boldsymbol{X}}(\alpha)}(x)
\end{aligned}
$$


Likewise, we can define the $\varepsilon$-quantile of $\widetilde{\boldsymbol{X}}, Q^{\varepsilon}(\widetilde{\boldsymbol{X}})$, as the minimum quantity that allows $F_{\widetilde{\boldsymbol{X}}}\left(Q^{\varepsilon}(\widetilde{\boldsymbol{X}})\right) \geq \varepsilon$, i.e. $Q^{\varepsilon}(\widetilde{\boldsymbol{X}})=F_{\widetilde{\boldsymbol{X}}}^{-1}(\varepsilon)$. Again, with the RVs $\underline{\boldsymbol{X}}(\alpha)$ and $\overline{\boldsymbol{X}}(\alpha)$ obtained from $\widetilde{\boldsymbol{X}}$,

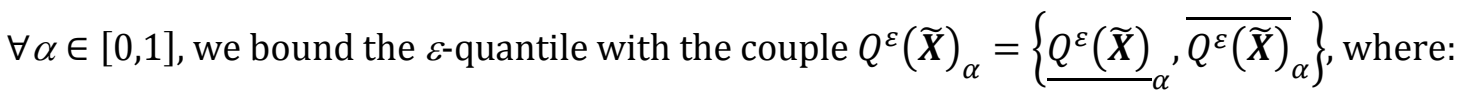

$$
\begin{aligned}
& \frac{Q^{\varepsilon}(\widetilde{\boldsymbol{X}})}{\alpha}=\left\{\min x \mid F_{\underline{\boldsymbol{X}}(\alpha)}(x) \geq \varepsilon\right\} \\
& \overline{Q^{\varepsilon}(\widetilde{\boldsymbol{X}})_{\alpha}}=\left\{\min x \mid F_{\overline{\boldsymbol{X}}(\alpha)}(x) \geq \varepsilon\right\}
\end{aligned}
$$

The mathematical expectation of a FRV is a FN $E(\widetilde{\boldsymbol{X}})$ whose $\alpha$-cuts are $E(\widetilde{\boldsymbol{X}})_{\alpha}=$ $[E(\underline{\boldsymbol{X}}(\alpha)), E(\overline{\boldsymbol{X}}(\alpha))]$. To obtain its defuzzified value (e.g. to rank FRVs from their mathematical expectations), López-Díaz and Gil (1998) propose using the concept of expected value of a FN, developed by Campos and González (1989), given its desirable properties for fuzzy decision problems. López-Díaz and Gil (1998) show that the fundamentals of the fuzzy utility function can be established by means of an axiomatic development of the fuzzy expected utility.

The variance measure of a FRV admits a fuzzy definition, in the same way as the expectation (Kruse and Meyer (1987)) or a real value (Körner (1997), Näther (2000) and Feng et al. (2001)). Following Couso and Dubois (2009) considerations, Andrés-Sánchez and González-Vila (2012) propose using the definition in Feng et al. (2001) for the pricing of life contingencies. In this paper the variance of $\widetilde{\boldsymbol{X}}, V(\widetilde{\boldsymbol{X}})$, is defined as:

$$
V(\widetilde{\boldsymbol{X}})=\frac{1}{2} \int_{0}^{1}[V(\underline{\boldsymbol{X}}(\alpha))+V(\overline{\boldsymbol{X}}(\alpha))] d \alpha
$$

where $V(\cdot)$ on RVs stands for the usual variance operator. Of course, the standard deviation is $S D(\widetilde{\boldsymbol{X}})=\sqrt{V(\widetilde{\boldsymbol{X}})}$.

Let us examine some results when the outcomes of the FRVs are STFNs, i.e., the $j$ th outcome is $\tilde{X}_{j}=\left(X_{j}, r_{X_{j}}\right)$. If we symbolize as $X$ the RV whose realizations are the centers of $\tilde{X}_{j}$, i.e. $X_{j}$, and as $\boldsymbol{r}_{X}$ the RV that can be built up from the spreads, $r_{X_{j}}, \forall j=1,2, \ldots, n$, the FRV $\widetilde{\boldsymbol{X}}$ can be represented as $\widetilde{\boldsymbol{X}}=\left(\boldsymbol{X}, \boldsymbol{r}_{\boldsymbol{X}}\right)$.

Example 1: Let us consider a probability space defined by $(\Omega, \mathcal{A}, P)$ where $\Omega$ is a discrete space of elementary events $\left\{\omega_{1}, \omega_{2}\right\}$. We define the FRV $\widetilde{\boldsymbol{X}}$ as follows:

$$
\begin{array}{ccc}
\widetilde{\boldsymbol{X}}: \Omega & \rightarrow & F(\Re) \\
\omega_{1} & \rightarrow & \widetilde{\boldsymbol{X}}\left(\omega_{1}\right)=(2,0.01)=\tilde{X}_{1} \\
\omega_{2} & \rightarrow & \widetilde{\boldsymbol{X}}\left(\omega_{2}\right)=(3,0.005)=\tilde{X}_{2}
\end{array}
$$

being the $\alpha$-cuts of these FNs, considering (1):

$$
X_{1_{\alpha}}=[2-0.01(1-\alpha), 2+0.01(1-\alpha)]
$$




$$
X_{2_{\alpha}}=[3-0.005(1-\alpha), 3+0.005(1-\alpha)]
$$

As explained above, $\widetilde{\boldsymbol{X}}$ defines, $\forall \alpha \in[0,1]$, an infima $\operatorname{RV} \underline{\boldsymbol{X}}(\alpha)$ and a suprema $\operatorname{RV} \overline{\boldsymbol{X}}(\alpha)$, whose realizations are, respectively, $\{2-0.01(1-\alpha), 3-0.005(1-\alpha)\}$ and $\{2+0.01(1-\alpha), 3+0.005(1-\alpha)\}$.

Furthermore, it is possible to define the RVs $\boldsymbol{X}$ and $\boldsymbol{r}_{\boldsymbol{X}}$, whose realizations are, respectively, $\{2,3\}$ and $\{0.01,0.05\}$ and, in this way, the FRV can be represented by $\widetilde{\boldsymbol{X}}=\left(\boldsymbol{X}, \boldsymbol{r}_{\boldsymbol{X}}\right)$.

From this representation of this FRV, whose outcomes are a STFN, it follows that the lower and upper RVs for a given $\alpha$-level are:

$$
\begin{aligned}
& \underline{\boldsymbol{X}}(\alpha)=\boldsymbol{X}-\boldsymbol{r}_{\boldsymbol{X}}(1-\alpha) \\
& \overline{\boldsymbol{X}}(\alpha)=\boldsymbol{X}+\boldsymbol{r}_{\boldsymbol{X}}(1-\alpha)
\end{aligned}
$$

As discussed above, the $\alpha$-cuts of the mathematical expectation of the FRV $\widetilde{\boldsymbol{X}}$ can be obtained from $E(\underline{\boldsymbol{X}}(\alpha))$ and $E(\overline{\boldsymbol{X}}(\alpha))$. Since $E(\underline{\boldsymbol{X}}(\alpha))=E(\boldsymbol{X})-E\left(\boldsymbol{r}_{\boldsymbol{X}}\right)(1-\alpha)$, with similar expression for $E(\overline{\boldsymbol{X}}(\alpha))$, it turns out that the mathematical expectation of $\widetilde{\boldsymbol{X}}$ is also a STFN with:

$$
E(\widetilde{\boldsymbol{X}})=\left(E(\boldsymbol{X}), E\left(\boldsymbol{r}_{\boldsymbol{X}}\right)\right)
$$

And consequently, considering, (2), the expected value of the mathematical expectation is:

$$
E V(E(\widetilde{\boldsymbol{X}}) ; \lambda)=E(\boldsymbol{X})+E\left(\boldsymbol{r}_{\boldsymbol{X}}\right)\left(\lambda-\frac{1}{2}\right)
$$

For the variance, from (7) we can write:

$$
\begin{aligned}
V(\widetilde{\boldsymbol{X}})=\frac{1}{2} \int_{0}^{1}\left[V\left(\boldsymbol{X}-\boldsymbol{r}_{\boldsymbol{X}}(1-\alpha)\right)+V\left(\boldsymbol{X}+\boldsymbol{r}_{\boldsymbol{X}}(1-\alpha)\right)\right] d \alpha= \\
=\frac{1}{2} \int_{0}^{1}\left\{E\left[\left(\boldsymbol{X}-\boldsymbol{r}_{\boldsymbol{X}}(1-\alpha)\right)^{2}-\left(E(\boldsymbol{X})-E\left(\boldsymbol{r}_{\boldsymbol{X}}\right)(1-\alpha)\right)^{2}\right]+\right. \\
\left.+E\left[\left(\boldsymbol{X}+\boldsymbol{r}_{\boldsymbol{X}}(1-\alpha)\right)^{2}-\left(E(\boldsymbol{X})+E\left(\boldsymbol{r}_{\boldsymbol{X}}\right)(1-\alpha)\right)^{2}\right]\right\} d \alpha=V(\boldsymbol{X})+\frac{1}{3} V\left(\boldsymbol{r}_{\boldsymbol{X}}\right)
\end{aligned}
$$

Example 2: Consider that in example 1 the probabilities are $p\left(\omega_{1}\right)=0.6$ and $p\left(\omega_{1}\right)=0.4$. The mathematical expectation of $\widetilde{\boldsymbol{X}}$ is $E(\widetilde{\boldsymbol{X}})=(2.4,0.008)$ and its variance $V(\widetilde{\boldsymbol{X}})=$ 0.240002 . 
Note that in this section, as well as throughout the paper, an RV is denoted with a bold letter and a FN with a non-bold letter with the superscript " $\sim$ ". Thus, a FRV is denoted with a bold and superscripted letter.

\section{PRESENT VALUE WITH SYMMETRICAL TRIANGULAR FUZZY NUMBERS}

In this section we describe how to compute the present value of a stream of cash flows when they and the interest rate are given by a STFN. Let us begin with the crisp set of cash flows $F_{1}, F_{2}, \ldots, F_{n} \geq 0$ whose maturities are $t_{1}, t_{2}, \ldots, t_{n}$ years, respectively. To value them we use the interest rate $i \geq 0$. If we denote by $F=\left(F_{1}, F_{2}, \ldots, F_{n}\right)$, the present value of $F$ is the function:

$$
P V(F, i)=\sum_{j=1}^{n} F_{j}(1+i)^{-t_{j}}
$$

with:

$$
\begin{aligned}
& \frac{\partial P V(F, i)}{\partial F_{j}}=(1+i)^{-t_{j}}>0 \\
& \frac{\partial P V(F, i)}{\partial i}=-\frac{\sum_{j=1}^{n} F_{j} t_{j}(1+i)^{-t_{j}}}{1+i}<0
\end{aligned}
$$

Of course, the Macaulay duration can also be understood as a function of $F$ and $i$ as:

$$
D(F, i)=\frac{\sum_{j=1}^{n} F_{j} t_{j}(1+i)^{-t_{j}}}{\sum_{j=1}^{n} F_{j}(1+i)^{-t_{j}}}=-\frac{\frac{\partial P V(F, i)}{\partial i}(1+i)}{P V(F, i)}
$$

i.e.:

$$
\frac{\partial P V(F, i)}{\partial i}=-P V(F, i) \frac{D(F, i)}{1+i}
$$

Financial mathematics with fuzzy parameters was developed in the late 1980s and early '90s by such authors as Kaufmann (1986), Buckley (1987) and Li Calzi (1990). The problem consists, primarily, in evaluating the basic expression of the present value (10) (and also the accumulated value function) when interest rate(s) and cash flows are FNs.

In our case, we evaluate the net present value of fuzzy cash flows $\widetilde{F}_{j}, j=1,2, \ldots, n$, where $\tilde{F}_{j}=\left(F_{j}, r_{F_{j}}\right)$ and the fuzzy interest rate $\tilde{\imath}=\left(i, r_{i}\right)$. Likewise, we denote as $\tilde{F}=$ $\left(\tilde{F}_{1}, \tilde{F}_{2}, \ldots, \tilde{F}_{n}\right), F_{C}=\left(F_{1}, F_{2}, \ldots, F_{n}\right)$ and $r_{F}=\left(r_{F_{1}}, r_{F_{2}}, \ldots, r_{F_{n}}\right)$. The fuzzy present value is the FN $\widetilde{P V}$ induced by $\tilde{F}$ and $\tilde{l}$ through the function $P V(\cdot)$, i.e. $\widetilde{P V}=P V(\widetilde{F}, \tilde{\imath})$. Its $\alpha$-cuts representation is, from (3): 


$$
\begin{aligned}
& P V_{\alpha}=[\underline{P V}(\alpha), \overline{P V}(\alpha)]= \\
& =\left[\sum_{j=1}^{n}\left[F_{j}-r_{F_{j}}(1-\alpha)\right]\left(1+i+r_{i}(1-\alpha)\right)^{-t_{j}}, \sum_{j=1}^{n}\left[F_{j}+r_{F_{j}}(1-\alpha)\right]\left(1+i-r_{i}(1-\alpha)\right)^{-t_{j}}\right]
\end{aligned}
$$

So, $\widetilde{P V}$ does not maintain the STFN shape. However, bearing (4) and (11) in mind, we can approximate it as $\widetilde{P V} \approx\left(P V\left(F_{C}, i\right), r_{P V}\right)$ where:

$$
\begin{aligned}
& P V\left(F_{C}, i\right)=\sum_{j=1}^{n} F_{C}(1+i)^{-t_{j}} \\
& r_{P V}=P V\left(r_{F}, i\right)+P V\left(F_{C}, i\right) \frac{D\left(F_{C}, i\right)}{1+i} r_{i}
\end{aligned}
$$

The above result is very appealing from a financial perspective. The most reliable value of the approximation for $\widetilde{P V}$ is obtained by evaluating the present value in the most possible values of cash flows and interest rates. The uncertainty of the present value, $r_{P V}$, comes from two sources: the uncertainty of cash flows and the spread of the interest rate. The contribution of cash flow spreads in the uncertainty of the approximation of $\widetilde{P V}$ is its own present value with the most reliable interest rate. Likewise, the fuzziness that comes from the interest rate is measured with the Macaulay duration, as is common in standard fixed income analysis.

Table 1 shows the $\alpha$-cuts of the present value of the cash flows $\widetilde{F}_{1}=(1000,50)$, $\tilde{F}_{2}=(1500,75), \tilde{F}_{3}=(2000,100)$ and $\tilde{F}_{4}=(2500,100)$, whose maturities are $1,2,3$ and 4 years, respectively. The interest rate is $\tilde{\imath}=(0.02,0.005)$. We define the errors:

$$
\begin{aligned}
& \underline{\operatorname{err}}(\alpha)=\frac{\left|\underline{P V}(\alpha)-\underline{P V^{\prime}}(\alpha)\right|}{\underline{P V}(\alpha)} \\
& \overline{\operatorname{err}}(\alpha)=\frac{\left|\overline{P V}(\alpha)-\overline{P V}^{\prime}(\alpha)\right|}{\overline{P V}(\alpha)}
\end{aligned}
$$

being $\underline{P V}(\alpha), \overline{P V}(\alpha)$ the extremes of the $\alpha$-cuts obtained with (12) and $\underline{P V}(\alpha)$ and $\overline{P V}^{\prime}(\alpha)$ these extremes in the STFN approximation of $\widetilde{P V}$ in (13). Notice that the error increases when $\alpha$ decreases because the Taylor expansion is developed from $\alpha=1$ to lower levels $(0 \leq \alpha<1)$. In any case, they are never greater than $0.1 \%$ and so, in our opinion, we can conclude that the proposed approximation is quite accurate.

Table 1. Comparison of the $\alpha$-cuts of the fuzzy present value and its STFN approximation

\begin{tabular}{|c|c|c|c|c|c|c|}
\cline { 2 - 5 } \multicolumn{1}{c|}{} & \multicolumn{3}{c|}{$\widetilde{P V}$} & \multicolumn{2}{c|}{$\left(P V\left(F_{C}, i\right), r_{P V}\right)$} & \multicolumn{2}{c|}{} \\
\hline$\alpha$ & $\underline{P V}(\alpha)$ & $\overline{P V}(\alpha)$ & $\underline{P V^{\prime}}(\alpha)$ & $\overline{P V^{\prime}}(\alpha)$ & $\underline{\operatorname{err}}(\alpha)$ & $\overline{\operatorname{err}}(\alpha)$ \\
\hline 1 & 6616.40 & 6616.40 & 6616.40 & 6616.40 & $0.00 \%$ & $0.00 \%$ \\
\hline 0,75 & 6516.80 & 6716.64 & 6516.49 & 6716.32 & $0.00 \%$ & $0.00 \%$ \\
\hline 0,5 & 6417.84 & 6817.52 & 6416.57 & 6816.24 & $0.02 \%$ & $0.02 \%$ \\
\hline 0,25 & 6319.50 & 6919.04 & 6316.65 & 6916.15 & $0.05 \%$ & $0.04 \%$ \\
\hline
\end{tabular}




\begin{tabular}{|l|l|l|l|l|l|l|}
\hline 0 & 6221.79 & 7021.22 & 6216.74 & 7016.07 & $0.08 \%$ & $0.07 \%$ \\
\hline
\end{tabular}

Now, let us symbolize the present value of a stream of unitary cash flows as $P V(i)=$ $\sum_{j=1}^{n}(1+i)^{-t_{j}}$ and its duration as $D(i)=\frac{\sum_{j=1}^{n} t_{j}(1+i)^{-t_{j}}}{P V(i)}$. Table 2 shows the expressions of the present value and the Macaulay duration for unitary financial and life insurance structures. Notice that Li and Panjer (1994) generalize the concept of duration under several yield rate regimes in life insurance pricing with deterministic mortality. Note that Table 2 aims to be general since pre-payable and deferred annuities embed any other kind of annuity. For example, the present value of a post-payable and immediate $n$-term annuity $a_{\bar{n} \mid i}$ can also be calculated as ${ }_{1} \ddot{a}_{\bar{n} \mid i}$. Likewise, whole life contracts are included by considering $n=\varpi-x+1$, with $\varpi$ the maximum attainable age in the mortality table, and immediate liabilities are also evaluated by taking $m=0$.

Table 2. Present value and duration of several unitary financial and life contingency structures

\begin{tabular}{|c|c|c|}
\hline & $\begin{array}{c}\text { Present value of unitary } \\
\text { payments }\end{array}$ & Macaulay duration of the unitary payments \\
\hline Discount factor & $d_{\bar{t} \mid i}=(1+i)^{-t}$ & $D\left(d_{\bar{t} \mid i}\right)=t$ \\
\hline $\begin{array}{l}\text { Post-payable and } \\
\text { immediate } n \text {-term } \\
\text { annuity }\end{array}$ & $a_{\bar{n} \mid i}=\frac{1-d_{\bar{n} \mid i}}{i}$ & $D\left(a_{\bar{n} \mid i}\right)=\frac{\left(1+\frac{1}{i}-n\right) a_{\bar{n} \mid i}-\frac{n}{i}}{a_{\bar{n} \mid i}}$ \\
\hline $\begin{array}{l}\text { Pre-payable and } \\
\text { deferred } m \text { years } n \text { - } \\
\text { term annuity }\end{array}$ & $\begin{array}{l}m \ddot{a}_{\bar{n} \mid i}=\sum_{t=m}^{m+n-1}(1+i)^{-t} \\
=a_{\bar{n} \mid i} d_{\overline{m-1} \mid i}\end{array}$ & $D\left({ }_{m} \ddot{a}_{\bar{n} \mid i}\right)=\frac{\left[\left(m+\frac{1}{i}-n\right) a_{\bar{n} \mid i}-\frac{n}{i}\right] d_{\overline{m-1} \mid i}}{{ }_{m} \ddot{a}_{\bar{n} \mid i}}$ \\
\hline $\begin{array}{l}n \text {-years pure } \\
\text { endowment for a } \\
\text { person aged } x\end{array}$ & $A_{\overline{x: n \mid i}}=d_{\bar{n} \mid i{ }_{n}} p_{x}$ & $D\left(A_{\frac{1}{x: n \mid i}}\right)=n$ \\
\hline $\begin{array}{l}n \text {-years life insurance } \\
\text { deferred } m \text { years for a } \\
\text { person aged } x\end{array}$ & ${ }_{m \mid n} A_{\bar{x} \mid i}=\sum_{t=m+1}^{m+n} d_{\bar{t}|i t-1|} q_{x}$ & $D\left({ }_{m \mid n} A_{\bar{x} \mid i}\right)=\frac{\sum_{t=m+1}^{m+n} t d_{\bar{t} \mid i t} A_{t-1 \mid} q_{x}}{m \mid n}$ \\
\hline $\begin{array}{l}n \text {-year mixed } \\
\text { endowment deferred } \\
m \text { years for a person } \\
\text { aged } x \\
\end{array}$ & ${ }_{m} A_{\overline{x: n \mid i}}=A_{\overline{x: m+n \mid i}}+{ }_{m \mid n} A_{\bar{x} \mid i}$ & $D\left({ }_{m} A_{\overline{x: n \mid i}}\right)=\frac{(m+n) d_{\overline{m+n} \mid i} m+n p_{x}+\sum_{t=m+1}^{m+n} t d_{\bar{t}|i t-1|} q_{x}}{{ }_{m} A_{\overline{x: n \mid} \mid i}}$ \\
\hline $\begin{array}{c}\text { Pre-payable and } \\
\text { deferred } m \text { years } n \text { - } \\
\text { term life annuity for a } \\
\text { person aged } x \\
\end{array}$ & ${ }_{m} \ddot{a}_{\overline{x: n \mid i}}=\sum_{t=m}^{m+n-1} d_{\bar{t} \mid i t} p_{x}$ & $D\left({ }_{m} \ddot{a}_{\overline{x: n \mid i}}\right)=\frac{\sum_{t=m}^{m+n-1} t d_{\bar{t} \mid i} p_{x}}{{ }_{m} \ddot{a}_{\overline{x: n \mid} \mid i}}$ \\
\hline
\end{tabular}

Notes: ${ }^{*}{ }_{n} p_{x}$ is the probability that the insured, aged $x$, survives at age $x+n$.

${ }^{* *}{ }_{n \mid} q_{x}$ is the probability that the insured, aged $x$, dies within the $n$th year.

Let us define the fuzzy unitary payment by means of a FN of "approximately 1 monetary unit (m.u.)", which will be symbolized as $\tilde{u}$. If it is a STFN, then $\tilde{u}=\left(1, r_{u}\right)$ where $r_{u} \leq 1$. Of course, we can generate any other fuzzy positive quantity by using any scalar $F \geq 0$, $\tilde{F}=F \tilde{u}$. In our opinion, fuzzy monetary amounts may be suitable for modeling situations in which they are linked to the evolution of an economic index or when we are evaluating 
not only claimed quantities, but also future expenses associated with them that are not known exactly (e.g. settlement costs). In this situation, fuzzy unitary cash-flows cover the case where the cash flows are "approximately constant". In any case, all the results can be extended to fuzzy variable cash-flows.

For a fuzzy interest rate $\tilde{\imath}=\left(i, r_{i}\right)$, the present value of the stream of fuzzy unitary quantities is $\widetilde{P V} \approx\left(P V(i), r_{P V}\right)$, where, considering (13):

$$
\begin{gathered}
P V(i)=\sum_{j=1}^{n}(1+i)^{-t_{j}} \\
r_{P V}=r_{u} P V(i)+P V(i) \frac{D(i)}{i} r_{i}=P V(i)\left(r_{u}+\frac{D(i)}{1+i} r_{i}\right)
\end{gathered}
$$

Next, we evaluate several classical financial and actuarial structures with fuzzy interest rates and payments. To do so, we use the results in Table 2, (4) and (14). Notice that the expressions contained in Table 2 use the classical equivalence principle for life contingency structures and, so, they reduce the probabilities of insured events to deterministic rates of occurrence. This is the approach used in Lemaire (1990), Ostaszewski (1993), Betzuen et al. (1997) and so evaluating life insurance and annuity contracts is reduced to evaluating (10) with fuzzy parameters as we have just shown above.

- For a fuzzy unitary payment $\tilde{u}=\left(1, r_{u}\right)$, where $r_{u} \leq 1$, and for an interest rate $\tilde{\imath}=\left(i, r_{i}\right)$ the discount factor, is:

$$
\tilde{d}_{\bar{t} \mid i}=\tilde{u}(1+\tilde{l})^{-t}
$$

and by using the STFN approximation $\tilde{d}_{\bar{t} \mid i} \approx\left(d_{\bar{t} \mid i}, r_{d_{\bar{t} \mid i}}\right)$

$$
\begin{gathered}
d_{\bar{t} \mid i}=(1+i)^{-t} \\
r_{d_{\bar{t} \mid i}}=d_{\bar{t} \mid i}\left(r_{u}+\frac{t}{1+i} r_{i}\right)
\end{gathered}
$$

- In the case of a pre-payable and deferred $m$ years $n$-term annuity the fuzzy present value ${ }_{m} \tilde{\ddot{a}}_{\bar{n} \mid i}$ is:

$$
{ }_{m} \tilde{\tilde{a}}_{\overline{n \mid i}}=\tilde{u} \sum_{t=m}^{m+n-1}(1+\tilde{\imath})^{-t}
$$

and it can be approximated by ${ }_{m} \tilde{\tilde{a}}_{\overline{n \mid i}} \approx\left({ }_{m} \ddot{a}_{\bar{n} \mid i}, r_{m} \ddot{a}_{\bar{n} \mid i}\right)$, where, by using (14):

$$
\begin{gathered}
{ }_{m} \ddot{a}_{\bar{n} \mid i}=\frac{1-d_{\bar{n} \mid i}}{i} d_{\overline{m-1} \mid i} \\
r_{m} \ddot{a}_{\bar{n} \mid i}={ }_{m} \ddot{a}_{\bar{n} \mid i}\left(r_{u}+\frac{D\left({ }_{m} \ddot{a}_{\bar{n} \mid i}\right)}{1+i} r_{i}\right)
\end{gathered}
$$


- The mathematical expectation of the present value of an $n$-year pure endowment for a person aged $x$ is:

$$
\tilde{A}_{\frac{1}{x: n \mid i}}=\tilde{d}_{\bar{n} \mid i n} p_{x}
$$

and $\tilde{A}_{\frac{1}{x: n \mid i}} \approx\left(A_{\frac{1}{x: n \mid i}, r_{A \frac{1}{x: n \mid i}}}\right)$, being:

$$
\begin{aligned}
& A_{\bar{x} \frac{1}{x: n \mid i}}=d_{\bar{n} \mid i n} p_{x} \\
& r_{\frac{1}{x: n \mid i}}=A_{\frac{1}{x: n \mid i}}\left(r_{u}+\frac{n}{1+i} r_{i}\right)
\end{aligned}
$$

- In the case of an $n$-year life insurance deferred $m$ years for a person aged $x$ and fuzzy unitary payments, the fuzzy mathematical expectation value is:

$$
{ }_{m \mid n} \tilde{A}_{\bar{x} \mid i}=\tilde{u} \sum_{t=m+1}^{m+n} \tilde{d}_{\bar{t}|i t-1|} q_{x}
$$

So, ${ }_{m \mid n} \tilde{A}_{\bar{x} \mid i} \approx\left({ }_{m \mid n} A_{\bar{x} \mid i}, r_{m \mid n} A_{\bar{x} \mid i}\right)$, where:

$$
\begin{array}{r}
m \mid n A_{\bar{x} \mid i}=\sum_{t=m+1}^{m+n} d_{\bar{t}|i t-1|} q_{x} \\
r_{m \mid n} A_{\bar{x} \mid i}={ }_{m \mid n} A_{\bar{x} \mid i}\left(r_{u}+\frac{D\left({ }_{m \mid n} A_{\bar{x} \mid i}\right)}{1+i} r_{i}\right)
\end{array}
$$

- In the case of an $n$-year mixed endowment deferred $m$ years for a person aged $x$, we have ${ }_{m} \tilde{A}_{\bar{x}: n \mid i}=\tilde{A}_{\overline{x: m+n \mid i}}+{ }_{m \mid n} \tilde{A}_{\bar{x} \mid i}$. So we have only to sum two SFTNs to obtain the approximation of ${ }_{m} \tilde{A}_{\bar{x}: n \mid i} \approx\left({ }_{m} A_{\bar{x}: n \mid i}, r_{m A_{\bar{x}: n \mid}}\right)$, with:

$$
\begin{gathered}
{ }_{m} A_{\bar{x}: \bar{n} \mid i}=A_{\overline{x: m+n \mid i}}+{ }_{m \mid n} A_{\bar{x} \mid i} \\
r_{m A_{\bar{x}: n \mid i}}=A_{\overline{x: m+n \mid i}}\left(r_{u}+\frac{m+n}{1+i} r_{i}\right)+{ }_{m \mid n} A_{\bar{x} \mid i}\left(r_{u}+\frac{D\left({ }_{m \mid n} A_{\bar{x} \mid i}\right)}{1+i} r_{i}\right)= \\
={ }_{m} A_{\overline{x: n} \mid i}\left(r_{u}+\frac{D\left({ }_{m} A_{\bar{x}: n \mid i}\right)}{1+i} r_{i}\right)
\end{gathered}
$$

- Finally, for a pre-payable and deferred $m$ years $n$-term life annuity for a person aged $x$, the fuzzy mathematical expectation value is:

$$
m \tilde{\tilde{a}}_{\overline{x: n \mid i}}=\sum_{t=m}^{m+n-1} \tilde{d}_{\bar{t} \mid i t} p_{x}
$$


And so, ${ }_{m} \tilde{\ddot{a}}_{\overline{x: n \mid i}} \approx\left({ }_{m} \ddot{a}_{\overline{x: n \mid i}}, r_{m} \ddot{a}_{\overline{x: n \mid i}}\right)$ where:

$$
\begin{array}{r}
m \ddot{a}_{\overline{x: n \mid} \mid i}=\sum_{t=m}^{m+n-1} d_{\bar{t} \mid i}{ }_{t} p_{x} \\
r_{m} \ddot{a}_{\overline{x: n \mid i}}={ }_{m} \ddot{a}_{\overline{x: n \mid} \mid i}\left(r_{u}+\frac{D\left({ }_{m} \ddot{a}_{\overline{x: n \mid} \mid}\right)}{1+i} r_{i}\right)
\end{array}
$$

Whereas Table 3a shows the fuzzy mathematical expectation for several types of life contingencies, Table $3 \mathrm{~b}$ shows its defuzzified value with the concept of expected value of a FN contained in (2).

Table 3a. Fuzzy mathematical expectation of the present value of several immediate life

\begin{tabular}{|c|c|c|c|c|c|c|c|c|c|}
\hline & \multicolumn{3}{|c|}{$F \cdot{ }_{m \mid n} \tilde{A}_{\bar{x} \mid i}$} & \multicolumn{3}{|c|}{$F \cdot{ }_{m} \tilde{A}_{\bar{\chi}: n \mid i}$} & \multicolumn{3}{|c|}{$F \cdot{ }_{m} \tilde{\tilde{a}}_{\overline{x: n \mid}}$} \\
\hline$x$ & $F \cdot{ }_{m \mid n} A_{\bar{x} \mid i}$ & $F \cdot r_{m \mid n} A_{x \mid i}$ & $D\left(m \mid n A_{\bar{x} \mid i}\right)$ & $F \cdot{ }_{m} A_{\overline{x: n} \mid i}$ & $F \cdot r_{m A_{\bar{x}: n \mid i}}$ & $D\left({ }_{m} A_{x: n \mid i}\right)$ & $F \cdot{ }_{m} \ddot{a}_{\bar{x}: n \mid i}$ & $F \cdot r_{m a_{\bar{x}: n \mid i}}$ & $D\left({ }_{m} \ddot{a}_{\overline{x: n \mid i}}\right)$ \\
\hline 25 & 326.01 & 94.09 & 54.79 & 820.61 & 56.57 & 9.98 & 34374.28 & 4646.69 & 24.47 \\
\hline 35 & 394.87 & 95.57 & 45.29 & 820.88 & 56.52 & 9.97 & 30863.17 & 3710.70 & 21.41 \\
\hline 45 & 476.24 & 93.68 & 36.05 & 821.94 & 56.31 & 9.90 & 26713.92 & 2789.71 & 18.19 \\
\hline 55 & 567.82 & 87.90 & 27.50 & 824.22 & 55.87 & 9.75 & 22042.86 & 1939.60 & 14.83 \\
\hline 65 & 668.34 & 77.70 & 19.64 & 828.74 & 55.00 & 9.46 & 16917.07 & 1203.92 & 11.38 \\
\hline 75 & 775.85 & 62.71 & 12.41 & 843.43 & 52.16 & 8.54 & 11433.78 & 624.45 & 7.97 \\
\hline 85 & 872.96 & 46.06 & 6.68 & 884.77 & 44.08 & 6.08 & 6482.19 & 259.88 & 4.94 \\
\hline 95 & 933.82 & 34.18 & 3.39 & 934.47 & 34.13 & 3.37 & 3367.70 & 100.54 & 2.71 \\
\hline
\end{tabular}
contingencies

Notes: $\quad * \tilde{\imath}=(0.02,0.005), \tilde{u}=(1,0.02)$ and $F=1000$ m.u. The life contingency probabilities are taken from the mortality tables of the Spanish population for both males and females in the year 2010 included in the Human Mortality Database (http://www.mortality.org), and obtained as explained in Wilmoth et al. (2007).

** In all cases, life contingencies are immediate, i.e. $m=0$ and both life insurance and annuities are whole life, i.e. $n=\varpi-x+1$. On the other hand, for the mixed endowment we have considered $n=$ 10 .

Table $3 \mathrm{~b}$. Expected value for the fuzzy mathematical expectation of the present value of life contingencies in Table $3 \mathrm{a}$

\begin{tabular}{|c|c|c|c|c|c|c|c|c|c|}
\cline { 2 - 10 } \multicolumn{1}{c|}{} & \multicolumn{2}{c|}{$E V\left(1000 \cdot{ }_{m \mid n} \tilde{A}_{\bar{x} \mid i} ; \lambda\right)$} & \multicolumn{2}{c|}{$E V\left(1000 \cdot{ }_{m} \tilde{A}_{\overline{x: n} \mid i} ; \lambda\right)$} & \multicolumn{3}{c|}{$E V\left(1000 \cdot{ }_{m} \tilde{\ddot{a}}_{\overline{x: n} \mid i} ; \lambda\right)$} \\
\hline$\chi \lambda 2$ & 0 & 0.5 & 1 & 0 & 0.5 & 1 & 0 & 0.5 & 1 \\
\hline 25 & 278.97 & 326.01 & 373.06 & 792.33 & 820.61 & 848.90 & 32050.94 & 34374.28 & 36697.63 \\
\hline 35 & 347.09 & 394.87 & 442.66 & 792.62 & 820.88 & 849.14 & 29007.82 & 30863.17 & 32718.52 \\
\hline 45 & 429.40 & 476.24 & 523.08 & 793.79 & 821.94 & 850.10 & 25319.07 & 26713.92 & 28108.78 \\
\hline 55 & 523.87 & 567.82 & 611.77 & 796.29 & 824.22 & 852.16 & 21073.06 & 22042.86 & 23012.66 \\
\hline 65 & 629.49 & 668.34 & 707.19 & 801.24 & 828.74 & 856.24 & 16315.11 & 16917.07 & 17519.03 \\
\hline 75 & 744.50 & 775.85 & 807.21 & 817.35 & 843.43 & 869.51 & 11121.56 & 11433.78 & 11746.01 \\
\hline 85 & 849.93 & 872.96 & 895.99 & 862.73 & 884.77 & 906.81 & 6352.25 & 6482.19 & 6612.13 \\
\hline 95 & 916.73 & 933.82 & 950.91 & 917.41 & 934.47 & 951.54 & 3317.43 & 3367.70 & 3417.97 \\
\hline
\end{tabular}




\section{PRICING LIFE CONTINGENCIES WITH FUZZY RANDOM VARIABLES}

In the previous section we calculated the present value of several life contingency structures by "fuzzifying" the classical equivalence principle. This approach has the disadvantage that all the information providing a complete statistical description of the present values of life contingent liabilities is lost. To avoid this, we propose adapting the stochastic approach to life insurance and annuities to the case of fuzzy unitary cash flows and fuzzy discount interest rate by using the concept of FRV. Our developments here are based on Andrés-Sánchez and González-Vila (2012, 2014).

Any kind of life contingency policy produces for the insurer a FRV present value of life contingencies, $m \widetilde{\boldsymbol{L}}_{\overline{\boldsymbol{x}: \boldsymbol{n} \mid \boldsymbol{i}} \boldsymbol{i}}$, whose realizations can be approximated by STFNs. Here $m$ symbolizes the number of deferred years, $n$ is the time horizon of the contract, $x$ is the age of the insured person and $i$ represents the most reliable discount rate. Following the developments in the previous sections, we can write ${ }_{m} \tilde{\boldsymbol{L}}_{\overline{x: n} \mid \boldsymbol{i}} \approx\left({ }_{m} \boldsymbol{L}_{\overline{x: n \mid i} \boldsymbol{i}}, \boldsymbol{r}_{m} \boldsymbol{L}_{\overline{x: n \mid i}}\right)$. Furthermore, we denote the $j$ th outcome of ${ }_{\boldsymbol{m}} \tilde{\boldsymbol{L}}_{\overline{\boldsymbol{x}: \boldsymbol{n} \mid \boldsymbol{i}}}$ as $\widetilde{P V}_{j}(i)=\left(P V_{j}(i), r_{P V_{j}(i)}\right)$, where $P V_{j}(i)$ denotes the present value of 1 m.u. or a unitary annuity whereas $r_{P V_{j}}$ is, from (14):

$$
r_{P V_{j}(i)}=P V_{j}(i)\left(r_{u}+\frac{D_{j}(i)}{1+i} r_{i}\right)
$$

Thus, we can find the mathematical expectation of the FRV present value of life contingencies as:

$$
E\left({ }_{m} \tilde{\boldsymbol{L}}_{\overline{x: n \mid i}}\right)=\left(E\left({ }_{m} \boldsymbol{L}_{\overline{x: n \mid i}}\right), E\left(\boldsymbol{r}_{{ }_{m} \boldsymbol{L}_{\bar{x}: \mathbf{n} \mid i}}\right)\right)
$$

where:

$$
\begin{gathered}
E\left({ }_{\boldsymbol{m}} \boldsymbol{L}_{\overline{\boldsymbol{x}: \boldsymbol{n} \mid \boldsymbol{i}}}\right)=\sum_{j} P V_{j}(i) p_{j} \\
E\left(\boldsymbol{r}_{\boldsymbol{m}_{\boldsymbol{x} \mathbf{x} \mid \mathbf{i}}}\right)=\sum_{j} r_{P V_{j}(i)} p_{j}=\sum_{j} P V_{j}(i)\left(r_{u}+\frac{D_{j}(i)}{1+i} r_{i}\right) p_{j}
\end{gathered}
$$

and $p_{j}$ is the probability of the $j$ th outcome.

For the variance of ${ }_{m} \tilde{\boldsymbol{L}}_{\overline{\boldsymbol{x}: \boldsymbol{n} \mid \boldsymbol{i}}}$, taking into account (9), we can obtain:

$$
V\left({ }_{m} \tilde{\boldsymbol{L}}_{\overline{x: n \mid i}}\right)=V\left({ }_{m} \boldsymbol{L}_{\overline{x: n} \mid \boldsymbol{i}}\right)+\frac{1}{3} V\left(\boldsymbol{r}_{{ }_{m} \boldsymbol{L}_{\overline{x: n} \mid i}}\right)
$$

being:

$$
V\left({ }_{\boldsymbol{m}} \boldsymbol{L}_{\overline{\boldsymbol{x}: \boldsymbol{n} \mid \boldsymbol{i}}}\right)=E\left(\left({ }_{\boldsymbol{m}} \boldsymbol{L}_{\overline{\boldsymbol{x}: \boldsymbol{n} \mid \boldsymbol{i}}}\right)^{2}\right)-\left(E\left({ }_{\boldsymbol{m}} \boldsymbol{L} \overline{\bar{x}: \boldsymbol{n} \mid \boldsymbol{i}}\right)\right)^{2}=\sum_{j}\left(P V_{j}(i)\right)^{2} p_{j}-\left(\sum_{j} P V_{j}(i) p_{j}\right)^{2}
$$


and:

$$
\begin{aligned}
& V\left(\boldsymbol{r}_{m} L_{\overline{x: n \mid i}}\right)=E\left(\left(\boldsymbol{r}_{m} L_{\overline{x: n \mid i}}\right)^{2}\right)-\left(E\left(\boldsymbol{r}_{m L_{\overline{x: n \mid i}}}\right)\right)^{2}= \\
& =\sum_{j}\left[P V_{j}(i)\left(r_{u}+\frac{D_{j}(i)}{1+i} r_{i}\right)\right]^{2} p_{j}-\left(\sum_{j} P V_{j}(i)\left(r_{u}+\frac{D_{j}(i)}{1+i} r_{i}\right) p_{j}\right)^{2}
\end{aligned}
$$

In a life insurance contract, the randomness derives from the maturity of the payment of the insured amounts. So, we will symbolize as ${ }_{m \mid n} \boldsymbol{t}_{\boldsymbol{x}}$ the discrete RV "payment maturity" for a life insurance contract that is deferred $m$ years, with a time horizon $n$ and for an insured aged $x$ years. If there is no insured money for some death ages, then the outcome of ${ }_{m \mid n} t_{x}$ is $\infty$ years. So, in any contract of this kind, the FRV ${ }_{m} \tilde{\boldsymbol{L}}_{\overline{\boldsymbol{x}: \boldsymbol{n} \mid \boldsymbol{i}} \boldsymbol{i}}$ can be expressed as:

$$
{ }_{m} \tilde{L}_{\overline{x: n \mid i}}=\tilde{d} \overline{m_{m \mid n} t_{x} \mid i}
$$

which in terms of a STFN approximation can be written as:

$$
{ }_{m} \tilde{L}_{\overline{x: n \mid i}} \approx\left({ }_{m} \boldsymbol{L}_{\overline{x: n \mid i}}, r_{m^{\prime} L_{\overline{x: n} \mid i}}\right)=\left(d \overline{{ }_{m \mid n} t_{x} \mid i}, d \overline{{ }_{m \mid n} \boldsymbol{t}_{x} \mid i}\left(r_{u}+\frac{m \mid n}{1+i} t_{x}\right)\right)
$$

Similarly, in a life annuity the randomness derives from the number of terms to be paid by the insurer. We symbolize as ${ }_{\boldsymbol{m} \mid \boldsymbol{n}} \boldsymbol{N}_{\boldsymbol{x}}$ the discrete RV "number of terms" for a deferred $m$ years $n$ - term life annuity, where $x$ is the age of the insured. In this case we can express ${ }_{m} \widetilde{\boldsymbol{L}}_{\overline{x: n \mid i}}$ as:

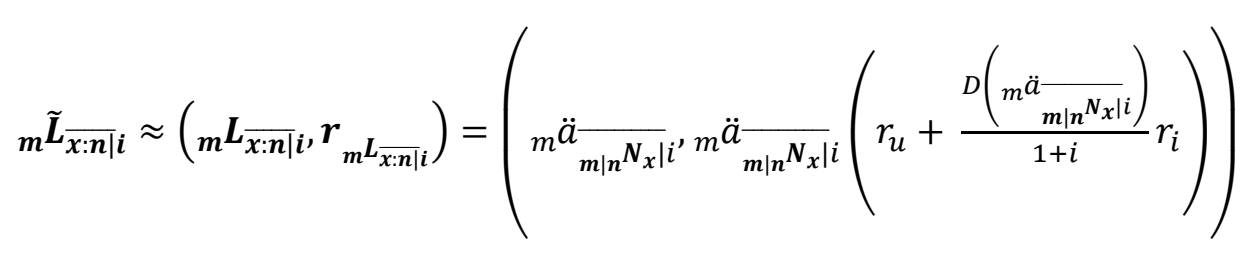

From this general setting, below we study some actuarial structures by applying the stochastic approach to life insurance and annuities to the case of fuzzy unitary cash flows and fuzzy discount interest rate.

- Let us consider an $n$-year pure endowment for a person aged $x$. In this case, it is supposed that the liability is $\tilde{u}$ m.u. if the insured survives $n$ years and no payment otherwise. From the mortality tables we can deduce the RV "payment maturity" $\boldsymbol{m} \mid \boldsymbol{n} \boldsymbol{t}_{\boldsymbol{x}}=\{n, \infty\}$ with probabilities $\left\{{ }_{n} p_{x},{ }_{n} q_{x}\right\}$. Hence, the FRV present value of the pure endowment, which we symbolize as $\widetilde{A}_{\frac{1}{x: n \mid i}}$, can be represented as the pair $\widetilde{\boldsymbol{A}}_{\frac{1}{\bar{x}: \boldsymbol{n} \mid i}}=$ $\left(\boldsymbol{A}_{\frac{1}{x: n \mid i}}, \boldsymbol{r}_{\frac{\boldsymbol{1}}{x: n \mid i}}\right)$ where, from (21): 


$$
\left(\boldsymbol{A}_{\frac{1}{x: n \mid i}}, \boldsymbol{r}_{\frac{A_{\bar{x}}}{x: n \mid i}}\right)=\left\{\begin{array}{ccc}
0 & \text { with probability } & { }_{n} q_{x} \\
\left(d_{\bar{n} \mid i}, d_{\bar{n} \mid i}\left(r_{u}+\frac{n}{1+i} r_{i}\right)\right) & \text { with probability } & { }_{n} p_{x}
\end{array}\right.
$$

So, $E\left(\widetilde{\boldsymbol{A}}_{\frac{1}{x: n \mid i}}\right)=\left(E\left(\boldsymbol{A}_{\frac{1}{x: n \mid i}}\right), E\left(\boldsymbol{r}_{\frac{A_{\frac{1}{x}}}{x: n \mid i}}\right)\right)=\tilde{A}_{\frac{1}{x: n \mid i}} \approx\left(A_{\frac{1}{x: n \mid i}}, r_{\frac{1}{x: n \mid i}}\right)$, i.e. (15a) and (15b).

Now, let us obtain the variance $V\left(\widetilde{\boldsymbol{A}}_{\frac{1}{x_{\mathbf{n} \mid i}}}\right)$. Following (9) it can be obtained as:

$$
V\left(\widetilde{A}_{\frac{1}{x: n \mid}}\right)=V\left(\boldsymbol{A}_{\frac{1}{x: n \mid i}}\right)+\frac{1}{3} V\left(\boldsymbol{r}_{\frac{1}{x: n \mid i}}\right)
$$

It is easy to show that:

$$
\begin{gathered}
V\left(\boldsymbol{A}_{\frac{1}{x: n \mid}}\right)=\left(d_{\bar{n} \mid i}\right)^{2}{ }_{n} p_{x}-\left(A_{\bar{x}: n \mid i}\right)^{2}=d_{\overline{2 n} \mid i n} p_{x n n} q_{x} \\
V\left(\boldsymbol{r}_{\frac{1}{x: n \mid i}}\right)=\left(r_{u}+\frac{n}{1+i} r_{i}\right)^{2} d_{\overline{2 n} \mid i n} p_{x}-\left(r_{\frac{1}{x: n \mid i}}\right)^{2}=\left(r_{u}+\frac{n}{1+i} r_{i}\right)^{2} d_{\overline{2 n} \mid i n} p_{x n} q_{x}
\end{gathered}
$$

and so:

$$
V\left(\widetilde{\boldsymbol{A}}_{\overline{\mathbf{x}} \mathbf{1} \mid}\right)=\left[1+\frac{1}{3}\left(r_{u}+\frac{n}{1+i} r_{i}\right)^{2}\right] d_{\overline{2 n} \mid i} p_{x} q_{x}
$$

- $\quad$ Now, let us take an $n$-year life insurance, deferred $m$ years, for a person aged $x$. Of course, if it were a whole life insurance policy, $n$ would be $\varpi-x+1$. The insured party aged $x$ will receive the STFN $\tilde{u}$ m.u. at the end of the year of his death, if he dies between the ages $x+m$ and $x+m+n$ and otherwise he does not receive anything. So, the RV

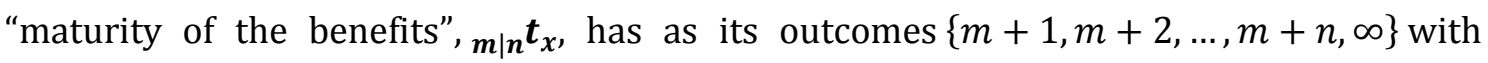

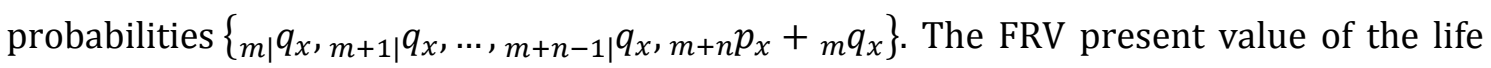
insurance, ${ }_{m \mid n} \widetilde{A}_{x}$, can be represented as ${ }_{m \mid n} \widetilde{A}_{\bar{x} \mid i}=\left({ }_{m \mid n} A_{\bar{x} \mid i}, r_{m \mid n} A_{\bar{x} \mid i}\right)$ where, from (21):

$$
\begin{aligned}
& \left({ }_{m \mid n} A_{\bar{x} \mid i}, r_{m \mid n} A_{\bar{x} \mid i}\right)= \\
& =\left\{\begin{array}{ccc}
0 & \text { with probability } & m+n p_{x}+{ }_{m} q_{x} \\
\left(d_{\bar{t} \mid i}, d_{\bar{t} \mid i}\left(r_{u}+\frac{t}{1+i} r_{i}\right)\right) & \text { with probability } & t-1 \mid q_{x}, t=m+1, m+2, \ldots, m+n
\end{array}\right.
\end{aligned}
$$

So, $E\left({ }_{m \mid n} \widetilde{A}_{\bar{x} \mid i}\right)=\left(E\left({ }_{m \mid n} A_{\bar{x} \mid i}\right), E\left(\boldsymbol{r}_{m \mid n} A_{\bar{x} \mid i}\right)\right)={ }_{m \mid n} \tilde{A}_{\bar{x} \mid i} \approx\left({ }_{m \mid n} A_{\bar{x} \mid i}, r_{m \mid n} A_{\bar{x} \mid i}\right)$ i.e. (16a) and (16b).

Considering (9), it turns out that: 


$$
V\left({ }_{m \mid n} \widetilde{A}_{\bar{x} \mid i}\right)=V\left({ }_{m \mid n} A_{\bar{x} \mid i}\right)+\frac{1}{3} V\left(r_{m \mid n} A_{\bar{x} \mid i}\right)
$$

and from the definition of ${ }_{\boldsymbol{m} \mid \boldsymbol{n}} \widetilde{\boldsymbol{A}}_{\overline{\boldsymbol{x}} \mid \boldsymbol{i}},(20)$ and Table 2 we find:

$$
\begin{gathered}
V\left({ }_{\boldsymbol{m} \mid \boldsymbol{n}} \boldsymbol{A}_{\overline{\mathbf{x}} \mid i}\right)=\sum_{t=m+1}^{m+n} d_{\overline{2 t}|i t-1|} q_{x}-\left({ }_{m \mid n} A_{\bar{x} \mid i}\right)^{2} \\
V\left(\boldsymbol{r}_{m \mid n} A_{\bar{x} \mid i}\right)=\sum_{t=m+1}^{m+n} d_{\overline{2 t} \mid i}\left(r_{u}+\frac{t}{1+i} r_{i}\right)^{2}{ }_{t-1 \mid} q_{x}-\left(r_{m \mid n} A_{\bar{x} \mid i}\right)^{2}
\end{gathered}
$$

- For an $n$-year mixed endowment, deferred $m$ years, linked to a person aged $x$ years, the outcomes of the RV "maturity of the benefits", $\boldsymbol{m | n} \boldsymbol{t}_{\boldsymbol{x}}$, are $\{\infty, m+1, m+$

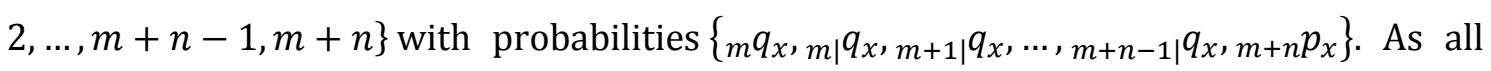
the benefits are considered as STFN, the fuzzy RV present value of liabilities, ${ }_{\boldsymbol{m}} \widetilde{\boldsymbol{A}} \overline{\bar{x}: \boldsymbol{n} \mid \boldsymbol{i}}$ can be

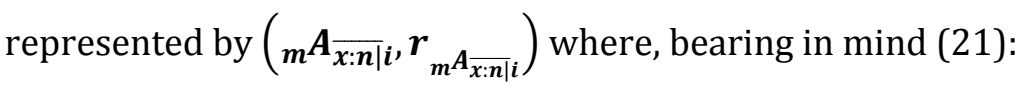

$$
\begin{aligned}
& \left({ }_{m} A_{\overline{x: n \mid i},}, r_{m} A_{\bar{x}: n \mid i}\right)= \\
& =\left\{\begin{array}{clc}
0 & \text { with probability } & m q_{x} \\
\left(d_{\bar{t} \mid i}, d_{\bar{t} \mid i}\left(r_{u}+\frac{t}{1+i} r_{i}\right)\right) & \text { with probability } & { }_{t-1 \mid} q_{x}, t=m+1, m+2, \ldots, m+n \\
\left(d_{\overline{m+n} \mid i}, d_{\overline{m+n} \mid i}\left(r_{u}+\frac{m+n}{1+i} r_{i}\right)\right) & \text { with probability } & { }_{m+n} p_{x}
\end{array}\right.
\end{aligned}
$$

In this case $E\left({ }_{m} \widetilde{A}_{\overline{x: n \mid i}}\right)=\left(E\left({ }_{m} A_{\overline{x: n \mid i}}\right), E\left(\boldsymbol{r}_{m} A_{\overline{x: n \mid i}}\right)\right)={ }_{m} \tilde{A}_{\overline{x: n \mid} i}$ so, considering (17), $E\left({ }_{\boldsymbol{m}} \boldsymbol{A}_{\overline{\boldsymbol{x}: n \mid i}}\right)={ }_{m} A_{\bar{x}: n \mid i}$ and $E\left(\boldsymbol{r}_{{ }_{m} A_{\bar{x}: n \mid i}}\right)=r_{m A_{\bar{x}: n \mid i}}$.

To obtain the variance $V\left({ }_{m} \widetilde{A}_{\overline{x: n \mid} \mid \boldsymbol{i}}\right)=V\left({ }_{m} \boldsymbol{A}_{\overline{x: n \mid} \mid \boldsymbol{i}}\right)+\frac{1}{3} V\left(\boldsymbol{r}_{{ }_{m} A_{\overline{x: n} \mid i}}\right)$, we have to take into account that, from (20):

$$
\begin{aligned}
& V\left({ }_{\boldsymbol{m}} \boldsymbol{A}_{\overline{\mathbf{x}: \boldsymbol{n} \mid \boldsymbol{i}}}\right)=\sum_{t=m+1}^{m+n} d_{\overline{2 t} \mid i} t-1 \mid q_{x}+d_{\overline{2(m+n)} \mid i}{ }_{m+n} p_{x}-\left({ }_{m} A_{\overline{x: n \mid} \mid}\right)^{2} \\
& V\left(\boldsymbol{r}_{m A_{\bar{x}: \mid i}}\right)=\sum_{t=m+1}^{m+n} d_{\overline{2 t} \mid i}\left(r_{u}+\frac{t}{1+i} r_{i}\right)^{2}{ }_{t-1 \mid} q_{x}+d_{\overline{2(m+n)} \mid i}\left(r_{u}+\frac{m+n}{1+i} r_{i}\right)_{m+n}^{2} p_{x}-\left(r_{m} A_{\bar{x}: n \mid i}\right)^{2}
\end{aligned}
$$

- $\quad$ Finally ${ }^{1}$, for a pre-payable, deferred $m$ years, $n$-term life annuity for a person aged $x$ the randomness will derive from the number of terms to be paid by the insurer. For this reason, the discrete RV "number of terms", ${ }_{m \mid n} \boldsymbol{N}_{\boldsymbol{x}}$, has to be considered. Its outcomes are

\footnotetext{
${ }^{1}$ Notice that an $n$-year pure endowment can be considered as a 1 -term annuity ${ }_{n} \widetilde{\widetilde{a}}_{\overline{x: 1 \mid i}}$ where the possible outcomes and probabilities of ${ }_{n \mid 1} N_{x}$ are $\{1,0\}$ and $\left\{{ }_{n} p_{x},{ }_{n} q_{x}\right\}$, respectively.
} 


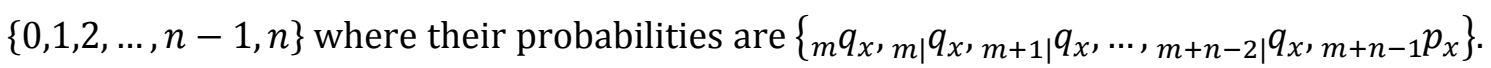
Once again, if we consider that the outcomes of the FRV present value of the annuity are STFN, it can be represented as ${ }_{m} \widetilde{\tilde{a}}_{\overline{x: n \mid i}}=\left({ }_{m} \ddot{a}_{\overline{x: n \mid i}}, \boldsymbol{r}_{m} \ddot{a}_{\overline{x: n \mid i}}\right)$.

Therefore, following (22):

$$
\begin{aligned}
& \left({ }_{m} \ddot{\boldsymbol{a}}_{\overline{x: n \mid i},}, \boldsymbol{r}_{{ }_{m} \ddot{a}_{\overline{x: n \mid i}}}\right)= \\
& =\left\{\begin{array}{ccc}
0 & \text { with probability } & m q_{x} \\
\left({ }_{m} \ddot{a}_{\bar{J} \mid i}, m \ddot{a}_{\bar{J} \mid i}\left(r_{u}+\frac{D\left({ }_{m} \ddot{a}_{\bar{J} \mid i}\right)}{1+i} r_{i}\right)\right) & \text { with probability } & m+j-1 \mid q_{x}, j=1,2, \ldots, n-1 \\
\left({ }_{m} \ddot{a}_{\bar{n} \mid i}, m \ddot{a}_{\bar{n} \mid i}\left(r_{u}+\frac{D\left({ }_{m} \ddot{a}_{\bar{n} \mid i}\right)}{1+i} r_{i}\right)\right) & \text { with probability } & m+n-1
\end{array}\right.
\end{aligned}
$$

Furthermore, $E\left({ }_{m} \widetilde{\ddot{a}}_{\overline{x: n \mid} \mid i}\right)={ }_{m} \tilde{\ddot{a}}_{\overline{x: n \mid i}} \approx\left({ }_{m} \ddot{a}_{\overline{x: n \mid} i}, r_{m} \ddot{a}_{\overline{x: n \mid i}}\right)$, where ${ }_{m} \ddot{a}_{\overline{x: n \mid i}}$ and $r_{m} \ddot{a}_{\bar{x}: n \mid i}$ can be obtained with the expressions contained in (18).

The determination of the variance requires considering the relation:

$$
V\left({ }_{m} \widetilde{\tilde{a}}_{\overline{x: n \mid i}}\right)=V\left({ }_{m} \ddot{\boldsymbol{a}}_{\overline{x: n \mid i}}\right)+\frac{1}{3} V\left(\boldsymbol{r}_{m} \ddot{a}_{\overline{x: n \mid i}}\right)
$$

where:

$$
\begin{gathered}
V\left({ }_{m} \ddot{\boldsymbol{a}}_{\overline{x: n \mid i}}\right)=\sum_{j=1}^{n-1}\left({ }_{m} \ddot{a}_{\bar{J} \mid i}\right)^{2}{ }_{m+j-1 \mid} q_{x}+\left({ }_{m} \ddot{a}_{\overline{n \mid i}}\right)^{2}{ }_{m+n-1} p_{x}-\left({ }_{m} \ddot{a}_{\overline{x: n \mid i}}\right)^{2} \\
V\left(\boldsymbol{r}_{m} \ddot{a}_{\bar{x}: n \mid i}\right)=\sum_{j=1}^{n-1}\left({ }_{m} \ddot{a}_{\bar{J} \mid i}\right)^{2}\left(r_{u}+\frac{D\left({ }_{m} \ddot{a}_{\bar{J} \mid i}\right)}{1+i} r_{i}\right)^{2}{ }_{m+j-1 \mid} q_{x}+ \\
\left.+{ }_{m} \ddot{a}_{\bar{n} \mid i}\right)^{2}\left(r_{u}+\frac{D\left({ }_{m} \ddot{a}_{\bar{n} \mid i}\right)}{1+i} r_{i}\right)^{2}{ }_{m+n-1} p_{x}-\left(r_{m} \ddot{a}_{\bar{x}: n \mid i}\right)^{2}
\end{gathered}
$$

\begin{tabular}{|c|c|c|c|c|c|c|c|c|c|}
\hline & \multicolumn{3}{|c|}{$1000 \cdot 0 \mid \omega-x+1=\widetilde{A}_{\bar{x} \mid i}$} & \multicolumn{3}{|c|}{$1000 \cdot{ }_{0} \widetilde{A}_{\overline{x: 10} \mid i}$} & \multicolumn{3}{|c|}{$1000 \cdot{ }_{0} \widetilde{\ddot{a}}_{\overline{x: \omega-x+1 \mid} i}$} \\
\hline$x$ & (1) & (2) & (3) & (1) & (2) & (3) & (1) & (2) & (3) \\
\hline 25 & 93.81 & 5.02 & 93.85 & 5.24 & 1.03 & 5.28 & 4782.24 & 1156.03 & 4828.59 \\
\hline 35 & 106.55 & 6.79 & 106.62 & 7.31 & 1.43 & 7.36 & 5432.41 & 1111.38 & 5470.17 \\
\hline 45 & 117.97 & 10.58 & 118.13 & 12.66 & 2.48 & 12.74 & 6016.22 & 1012.33 & 6044.55 \\
\hline 55 & 120.98 & 14.05 & 121.25 & 19.70 & 3.86 & 19.83 & 6170.71 & 844.53 & 6189.95 \\
\hline 65 & 113.97 & 16.11 & 114.35 & 28.31 & 5.55 & 28.49 & 5815.99 & 632.11 & 5827.43 \\
\hline 75 & 98.85 & 16.48 & 99.31 & 43.01 & 8.45 & 43.29 & 5046.75 & 411.42 & 5052.34 \\
\hline
\end{tabular}

Table 4 shows the standard deviation of the present value of the same life contingencies as those presented in Table 3a.

Table 4. Standard deviation of the present value of life contingencies in Table 3a 


\begin{tabular}{|l|c|c|c|c|c|c|c|c|c|}
\hline 85 & 73.01 & 13.83 & 73.44 & 55.32 & 11.02 & 55.69 & 3738.57 & 217.17 & 3740.67 \\
\hline 95 & 46.29 & 8.94 & 46.58 & 42.40 & 8.72 & 42.70 & 2238.69 & 93.88 & 2239.35 \\
\hline
\end{tabular}

Notes: * Life contingencies and technical basis are the same as in Table 3a. So, the mathematical expectation for the present value of liabilities is contained in this table.

** (1) stands for the standard deviation of the RV defined by the centers, (2) for the standard deviation of the RVs whose outcomes are the spreads and (3) is the standard deviation of the FRV present value.

\section{PRICING PORTFOLIOS OF FUZZY UNITARY LIFE CONTINGENCIES}

Computing the mathematical expectation and the variance for the whole portfolio, under the hypothesis of independence of mortality between policies is straightforward, given that the mathematical expectation and the variance of FRVs have similar properties to analogous indicators for conventional RVs.

If we symbolize the present value of the $j$ th liability of the portfolio as $\boldsymbol{m}_{\boldsymbol{j}} \tilde{\boldsymbol{L}}_{\overline{\boldsymbol{j}_{j}: \boldsymbol{n}_{\boldsymbol{j}} \mid \boldsymbol{i}}}=$ $\left({ }_{m_{j}} \boldsymbol{L}_{\overline{x_{j}: \boldsymbol{n}_{j} \mid i}}, \boldsymbol{r}_{m_{j} \boldsymbol{L}_{\overline{x_{j}: \boldsymbol{n}_{j} \mid i}}}\right)$, the present value of a portfolio made up of $J$ contracts is a FRV $\tilde{\boldsymbol{L}}$ where:

$$
\tilde{\boldsymbol{L}}=\sum_{j=1}^{J} \boldsymbol{m}_{j} \tilde{\boldsymbol{L}}_{\overline{x_{\jmath}: n_{\jmath} \mid} \boldsymbol{i}}
$$

And so, $\tilde{\boldsymbol{L}}=\left(\boldsymbol{L}, \boldsymbol{r}_{\boldsymbol{L}}\right)$ with:

$$
\left(\boldsymbol{L}, \boldsymbol{r}_{\boldsymbol{L}}\right)=\left(\sum_{j=1}^{J} \boldsymbol{m}_{j} \boldsymbol{L}_{\overline{x_{j}: n_{j} \mid i}}, \sum_{j=1}^{J} \boldsymbol{r}_{m_{j}} \boldsymbol{L}_{\overline{x_{J}}: n_{j} \mid i}\right)
$$

The rules for the expectation of RVs are identical to those for FRV. So, for $E(\tilde{\boldsymbol{L}})=$ $\left(E(\boldsymbol{L}), E\left(\boldsymbol{r}_{\boldsymbol{L}}\right)\right)$ we immediately derive:

$$
\begin{gathered}
E(\boldsymbol{L})=\sum_{j=1}^{J} E\left(\boldsymbol{m}_{j} \boldsymbol{L}_{\overline{x_{j}: \boldsymbol{n}_{\boldsymbol{j}} \mid i}}\right) \\
E\left(\boldsymbol{r}_{\boldsymbol{L}}\right)=\sum_{j=1}^{J} E\left(\boldsymbol{r}_{\left.\boldsymbol{m}_{j} \boldsymbol{L}_{\overline{x_{j}: n_{j} \mid i}}\right)}\right)
\end{gathered}
$$

Likewise, given that we are dealing with independent FRVs, to obtain $V(\tilde{\boldsymbol{L}})$ we have to compute:

$$
V(\boldsymbol{L})=\sum_{j=1}^{J} V\left({ }_{m_{j}} \boldsymbol{L}_{\overline{x_{j}: \boldsymbol{n}_{\boldsymbol{J}}} \mid i}\right)
$$




$$
V\left(\boldsymbol{r}_{L}\right)=\sum_{j=1}^{J} V\left(\boldsymbol{r}_{m_{j} L_{\overline{x_{j}: n_{j} \mid i}}}\right)
$$

And so, $V(\tilde{\boldsymbol{L}})=V(\boldsymbol{L})+\frac{1}{3} V\left(\boldsymbol{r}_{\boldsymbol{L}}\right)$.

On the other hand, under our hypothesis that the cost of claims and discount rates are STFN, we can extend the results in Andrés-Sánchez and González-Vila $(2012,2014)$ to compute the quantiles of the present value of liabilities. We should emphasize that these indicators play an important role in evaluating the solvency of an insurance company.

If the group of policies can be divided into large homogeneous sets of liabilities, a suitable way to approach $\tilde{\boldsymbol{L}}$ is by using a fuzzy normal RV with a fuzzy mean $E(\tilde{\boldsymbol{L}})=$ $\left(E(\boldsymbol{L}), E\left(\boldsymbol{r}_{\boldsymbol{L}}\right)\right)$ and a crisp standard deviation $S D(\tilde{\boldsymbol{L}})$. Fuzzy Gaussian RVs have been used in the financial context for portfolio selection (Inuiguchi and Ramik (2000)) and to obtain value-at-risk in a fuzzy environment (Zmeskal (2005)). In an actuarial context, Shapiro (2009) suggests their use in practical applications.

When the set of liabilities cannot be divided into broad groups of homogeneous policies, Andrés-Sánchez and González-Vila $(2012,2014)$ propose using statistical simulation to obtain empirically the FRV "present value of the portfolio's life contingencies". In these papers, the authors adapt the schema proposed in Pitacco (1986) to the fact that the outcomes are FNs. The steps that have to be taken to obtain the percentiles of the present value are presented below.

Step 1 Simulate $S$ times for each of the $J$ policies the $\mathrm{RV}{ }_{\boldsymbol{m} \mid \boldsymbol{n}} \boldsymbol{t}_{\boldsymbol{x}}$ in insurance contracts and $\boldsymbol{m} \mid \boldsymbol{n} \boldsymbol{N}_{\boldsymbol{x}}$ in annuity policies.

Step 2. By applying (23), (24), (25) or (26) to the sth simulation of the $j$ th life contingency contract, we obtain a STFN that quantifies the present value of this policy in the $s$ th simulation. Notice that the use of our STFN extension reduces notably the calculations that have to be implemented to obtain simulated fuzzy present values respect to AndrésSánchez and González-Vila $(2012,2014)$. In this paper, the quantification of the present value that derives from the $s$ th simulation for the $j$ th policy only requires the calculation of one crisp present value and a variability margin closely linked to the Macaulay duration of this present value. On the other hand, in those papers, given that the present value calculations are based on the $\alpha$-cuts of discount rates, if we take a not particularly detailed scale for $\alpha$ (specifically $\alpha=0,0.25,0.5,0.75,1$ ), to fit the present value of one concrete simulation would require calculating 10 values (the 10 extremes of $5 \alpha$-cuts). Calculating $\alpha$-cuts with a more detailed scale would clearly imply an increase in the number of calculations.

Step 3. The sth simulation of the whole portfolio of life contingencies is obtained as a STFN by summing the present value of the $J$ policies for that $s$ th simulation. So, we have an approximation to the FRV present value of the portfolio, $\tilde{\boldsymbol{L}}$, where each outcome has the same probability $1 / S$. 
Step 4. From the $\alpha$-cut representation of the approximation to $\tilde{\boldsymbol{L}}$ we can obtain its couple of the distribution functions with (5a) and (5b) and the bounds of their quantiles by using (6a) and (6b).

Tables $5 \mathrm{a}$ and $5 \mathrm{~b}$ describe a small set of immediate whole life insurances and show the 99th and $95^{\text {th }}$ percentiles of the present value of liabilities for segregated and aggregate subsets of contracts. Likewise, in Tables $6 \mathrm{a}$ and $6 \mathrm{~b}$ we develop an analogous numerical application for a set of life annuities.

Table 5a. Portfolio of whole life insurances $1000 \cdot 0 \mid \omega-x+1 \widetilde{A}_{\bar{x} \mid i}$ with several ages and contracts

\begin{tabular}{|c|c|c|c|c|}
\cline { 3 - 5 } \multicolumn{2}{c|}{} & \multicolumn{3}{c|}{$\tilde{\boldsymbol{L}}$} \\
\hline Age & $\begin{array}{c}\text { Number of } \\
\text { contracts }\end{array}$ & $E(\boldsymbol{L})$ & $E\left(\boldsymbol{r}_{\boldsymbol{L}}\right)$ & $S D(\tilde{\boldsymbol{L}})$ \\
\hline 55 & 5 & 2839.09 & 439.52 & 271.12 \\
\hline 65 & 10 & 6683.37 & 776.95 & 361.61 \\
\hline 75 & 5 & 3879.23 & 313.53 & 222.06 \\
\hline Whole portfolio & 25 & 13401.70 & 1530.00 & 503.57 \\
\hline
\end{tabular}

Note: $\quad *$ Whole life insurances and technical basis are the same as those considered in Tables 3a and 4.

Table 5b. 99th and 95th percentiles of portfolio and sub-portfolios of whole life insurances $1000 \cdot{ }_{0 \mid \omega-x+1} \widetilde{A}_{\bar{x} \mid i}$ in Table 5a

\begin{tabular}{|c|c|c|c|c|c|c|c|c|}
\hline & \multicolumn{4}{|c|}{$\begin{array}{l}\text { Contract: } 1000 \cdot{ }_{0} \mid \omega-\mathbf{5 5 + 1} \widetilde{A}_{\overline{55} \mid i} \\
\text { Number of contracts: } 5\end{array}$} & \multicolumn{4}{|c|}{$\begin{array}{l}\text { Contract: } 1000 \cdot \mathbf{0} \mid \omega-\mathbf{6 5 + 1} \widetilde{A}_{\overline{\mathbf{6 5}} \mid \boldsymbol{i}} \\
\quad \text { Number of contracts: } 10\end{array}$} \\
\hline$\alpha$ & 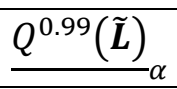 & $\overline{Q^{0.99}(\tilde{\boldsymbol{L}})_{\alpha}}$ & 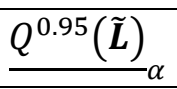 & $\overline{Q^{0.95}(\tilde{\boldsymbol{L}})_{\alpha}}$ & $Q^{0.99}(\tilde{\boldsymbol{L}})$ & $\overline{Q^{0.99}(\tilde{\boldsymbol{L}})_{\alpha}}$ & 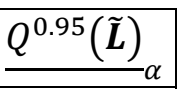 & $\overline{Q^{0.95}(\tilde{\boldsymbol{L}})_{\alpha}}$ \\
\hline 1 & 3577.59 & 3577.59 & 3323.50 & 3323.50 & 7637.49 & 7637.49 & 7347.51 & 7347.51 \\
\hline 0.75 & 2.02 & 52.01 & 29.71 & 7.30 & 7476.46 & 8.52 & 75.04 & 7519.98 \\
\hline 0.5 & 5.46 & 0.0 & 4.78 & & & 9.54 & 2.58 & 2.58 \\
\hline 0.25 & & & & & & & & 5.34 \\
\hline \multirow[t]{2}{*}{0} & 3237.87 & 13.64 & 2940.93 & 3698.41 & 6993.52 & 81.59 & 6655.65 & 8038.11 \\
\hline & \multicolumn{4}{|c|}{$\begin{array}{l}\text { Contract: } 1000 \cdot 0 \mid \omega-75+1 \widetilde{A}_{\overline{75} \mid \boldsymbol{i}} \\
\text { Number of contracts: } 5\end{array}$} & \multicolumn{4}{|c|}{ Whole portfolio } \\
\hline$\alpha$ & 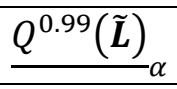 & $\overline{Q^{0.99}(\tilde{\boldsymbol{L}})_{\alpha}}$ & 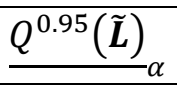 & $\overline{Q^{0.95}(\tilde{\boldsymbol{L}})_{\alpha}}$ & 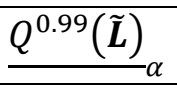 & $\overline{Q^{0.99}(\tilde{\boldsymbol{L}})_{\alpha}}$ & ${\frac{Q^{0.95}(\tilde{\boldsymbol{L}})}{\alpha}}$ & $\overline{Q^{0.95}(\tilde{\boldsymbol{L}})_{\alpha}}$ \\
\hline 1 & 4379.39 & 79.39 & 4227.66 & 4227.66 & 14911.43 & 14911.43 & 14477.85 & 14477.85 \\
\hline 0.75 & 4323.04 & 36.62 & 4165.48 & 4292.05 & 14580.40 & 15242.46 & 14131.10 & 14824.65 \\
\hline 0.5 & 4266.54 & 4493.85 & 4103.80 & 4356.44 & 14249.40 & 15573.49 & 13784.57 & 15173.82 \\
\hline 0.25 & 4209.45 & 1.09 & 4041.73 & 4420.77 & 13918.41 & 15904.52 & 3432.80 & 15525.92 \\
\hline 0 & 4153.06 & 4608.32 & 3978.06 & 4482.84 & 13590.51 & 16235.54 & 13082.85 & 15877.63 \\
\hline
\end{tabular}

Note: * Whole life insurances and technical basis are the same as those considered in Tables 3a and 4. 
Table 6a. Portfolio of whole life insurances $1000 \cdot{ }_{0} \widetilde{\ddot{a}}_{\overline{x: \omega-x+1 \mid i}}$ with several ages and contracts

\begin{tabular}{|c|c|r|r|c|}
\cline { 3 - 5 } \multicolumn{2}{c|}{} & \multicolumn{3}{c|}{$\tilde{\boldsymbol{L}}$} \\
\hline Age & $\begin{array}{c}\text { Number of } \\
\text { contracts }\end{array}$ & $E(\boldsymbol{L})$ & $E\left(\boldsymbol{r}_{\boldsymbol{L}}\right)$ & $S D(\tilde{\boldsymbol{L}})$ \\
\hline 55 & 5 & 110214.28 & 9698.01 & 13841.14 \\
\hline 65 & 10 & 169170.68 & 12039.18 & 18427.94 \\
\hline 75 & 5 & 57168.89 & 3122.25 & 11297.37 \\
\hline Whole portfolio & 25 & 57168.89 & 3122.25 & 25667.04 \\
\hline
\end{tabular}

Note: $\quad *$ Whole life insurances and technical basis are the same as those considered in Tables 3a and 4.

Table $6 \mathrm{~b} .99^{\text {th }}$ and $95^{\text {th }}$ percentiles of portfolio and sub-portfolios of whole life annuities $1000 \cdot{ }_{0} \widetilde{\ddot{a}}_{\overline{x: \omega-x+1 \mid i}}$ in Table 6a

Contract: $1000 \cdot{ }_{0} \widetilde{\tilde{\boldsymbol{a}}}_{\overline{55: \omega-55+1 \mid} \boldsymbol{i}}$

Number of contracts: 5

Contract: $1000 \cdot{ }_{0} \widetilde{\tilde{a}}_{\overline{65: \omega-65+1 \mid} i}$ Number of contracts: 10

\begin{tabular}{|c|c|c|c|c|c|c|c|c|}
\hline$\alpha$ & ${\frac{Q^{0.99}(\tilde{\boldsymbol{L}})}{\alpha}}$ & $Q^{0.99}(\tilde{\boldsymbol{L}})_{\alpha}$ & $Q^{0.95}(\tilde{\boldsymbol{L}})$ & $Q^{0.95}(\tilde{\boldsymbol{L}})_{\alpha}$ & $Q^{0.99}(\tilde{\boldsymbol{L}})$ & $Q^{0.99}(\tilde{\boldsymbol{L}})_{\alpha}$ & $Q^{0.95}(\tilde{\boldsymbol{L}})$ & $\overline{Q^{0.95(\tilde{\boldsymbol{L}})_{\alpha}}}$ \\
\hline 1 & 136297.47 & 136297.47 & 130500.05 & 130500.05 & 213630.32 & 213630.32 & 199509.08 & 199509.08 \\
\hline 0.75 & 132853.68 & 139741.26 & 127345.77 & 133665.07 & 209360.34 & 217900.30 & 195620.09 & 203300.79 \\
\hline 0.5 & 129409.88 & 143185.06 & 124191.48 & 136881.81 & 205090.36 & 222170.28 & 191769.42 & 207092.50 \\
\hline 0.25 & 125966.09 & 46628.85 & 121037.20 & 140098.56 & 200820.38 & 226440.26 & 187962.84 & 210884.20 \\
\hline \multirow[t]{2}{*}{0} & 122522.30 & 150072.64 & 117882.91 & 143311.54 & 196550.40 & 230710.24 & 184156.64 & 214675.91 \\
\hline & \multicolumn{4}{|c|}{$\begin{array}{c}\text { Contract: } 1000 \cdot{ }_{0} \widetilde{\tilde{\boldsymbol{a}}}_{\overline{75: \omega-75+1 \mid} \boldsymbol{i}} \\
\text { Number of contracts: } 5 \\
\end{array}$} & \multicolumn{4}{|c|}{ Whole portfolio } \\
\hline$\alpha$ & 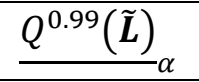 & $\overline{Q^{0.99}(\tilde{\boldsymbol{L}})_{\alpha}}$ & 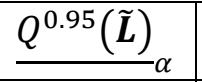 & $\overline{Q^{0.95}(\tilde{\boldsymbol{L}})_{\alpha}}$ & 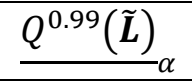 & $\overline{Q^{0.99}(\tilde{\boldsymbol{L}})_{\alpha}}$ & $Q^{0.95}(\tilde{\boldsymbol{L}})$ & $\overline{Q^{0.95}(\tilde{\boldsymbol{L}})_{\alpha}}$ \\
\hline 1 & 82582.50 & 82582.50 & 75146.83 & 75146.83 & 332116.81 & 332116.81 & 316496.67 & 316496.67 \\
\hline 0.75 & 81276.75 & 83888.26 & 74014.93 & 76278.78 & 325290.95 & 338942.66 & 310049.54 & 322946.50 \\
\hline 0.5 & 79970.99 & 85194.01 & 72884.71 & 77410.72 & 318465.10 & 345768.52 & 303651.51 & 329399.04 \\
\hline 0.25 & 78665.57 & 86499.77 & 71754.97 & 78544.03 & 311639.24 & 352594.37 & 297136.33 & 335848.24 \\
\hline 0 & 77360.20 & 87805.53 & 70625.22 & 79678.22 & 304716.27 & 359420.23 & 290618.21 & 342250.14 \\
\hline
\end{tabular}

Note: $\quad *$ Whole life insurances and technical basis are the same as those considered in Tables 3a and 4

\section{CONCLUSIONS}

In this paper we have extended the results reported in Andrés-Sánchez and González-Vila $(2012,2014)$, which model life contingency pricing with fuzzy random variables (FRVs), in two ways. First, we allow not only the discount rate(s), but also the amounts to be paid out by the insurance companies, to be fuzzy. Thus, our schema can be used when part of the cost of an insurance policy is not known with precision (e.g. when insured amounts are indexed or when future expenses such as settlement costs need to be taken into account). Second, under the hypothesis that the parameters are symmetrical triangular fuzzy numbers (STFNs), we have obtained several indicators that enable us to obtain the fair (or 
expected) price of life contingencies, the reasonable variability of this mathematical expectation and the variability of the present cost of these life contingencies (i.e. the solvency cost).

Using the STFN approximation based on Dubois and prade $(1980,1993)$ to the present value with STFN parameters enable us to obtain interesting operational expressions that simplify the computational requirements of the results in Andrés-Sánchez and GonzálezVila $(2012,2014)$. In fact, the calculation of the fuzzy present value is reduced to obtain the most reliable value with the centers of interest rate and cash flows. Likewise, the variability of the present value is intuitively (from a financial perspective) evaluated, by aggregating the present value of cash flow spreads and the variability that comes from interest rate uncertainty, which is linearly approximated, as is usual in standard financial mathematics, with the Macaulay duration. The use of FRVs with STFN outcomes allows us to describe the present value of life contingencies using just two conventional random variables: the centers and the spreads (i.e. the uncertainty) of the present value of insured amounts. Thus, the calculation of such magnitudes as mathematical expectation, standard deviation, quantiles, etc. for a contract (and, consequently, for a portfolio of policies) is relatively easy and intuitive.

The results presented in this paper can, we believe, be readily extended to more general forms of fuzzy numbers. Moreover, in relation to life insurance policies, a slight generalization of our proposed developments can be derived from the consideration that the time when the insured amount is paid is not the end of the year of death, but any moment within that year and so, the payment maturity date is fuzzy. The latter may depend, for example, on the exact date of death, the delay before the heirs file their claim, or other factors. For example, if the insured dies in the $r$ th year, the maturity payment may be the STFN $\left(r+\frac{1}{2}, \frac{1}{2}\right)$, i.e. it is recognized that payment of the insured amount can be made at the beginning of the $r$ th year or at the end of that year with the most reliable value being at the midpoint. Other natural ways in which our findings can be extended include the use of the fuzzy, non-flat, temporal structure of interest rates, as proposed in Ostaszewski (1993) and in Andrés-Sánchez and Terceño (2003), or the introduction of fuzzy uncertainty in the future lifetime, as suggested Shapiro (2013).

\section{REFERENCES}

1. Andrés-Sánchez, J. de (2014). Fuzzy claim reserving in non-life insurance. Computational. Science and Information Systems, 11 (2), 825-838. doi:10.2298/CSIS121225045A

2. Andrés-Sánchez, J. de and González-Vila Puchades, L. (2012). Using fuzzy random variables in life annuities pricing. Fuzzy Sets and Systems, 188, 27-44. doi:10.1016/j.fss.2011.05.024

3. Andrés-Sánchez, J. de and González-Vila Puchades, L. (2014). Pricing endowments with soft computing. Economic Computation and Economic Cybernetics Studies Research, 1, 124-142. 
4. Andrés-Sánchez, J. de and Terceño, A. (2003). Applications of Fuzzy Regression in Actuarial Analysis. Journal of Risk and Insurance, 70, 665-699. doi:10.1046/j.00224367.2003.00070.x

5. Betzuen, A., Jiménez, M. and Rivas, J. A. (1997). Actuarial mathematics with fuzzy parameters. An application to collective pension plans. Fuzzy Economic Review, 2 (2), 47-66.

6. Buckley, J.J. (1987). The fuzzy mathematics of finance. Fuzzy Sets and Systems, 21, 257273. doi:10.1016/0165-0114(87)90128-X

7. Buckley, J.J. and Qu, Y. (1990). On using $\alpha$-cuts to evaluate fuzzy equations. Fuzzy Sets and Systems, 38, 309-312. doi:10.1016/0165-0114(90)90204-J

8. Campos, L.M. and González, A. (1989). A subjective approach for ranking fuzzy numbers. Fuzzy Sets and Systems, 29, 145-153. doi:10.1016/0165-0114(89)90188-7.

9. Couso, I. and Dubois, D. (2009). On the variability of the concept of variance for fuzzy random variables. Fuzzy Systems, IEEE Transactions, 17 (5), 1070-1080. doi:10.1109/TFUZZ.2009.2021617

10. Cummins, J.D. and Derrig, R.A. (1997). Fuzzy financial pricing of property-liability insurance. North American Actuarial Journal, 1, 21-44. doi:10.1080/10920277.1997.10595640

11. De Wit, G.W. (1982) Underwriting and uncertainty. Insurance: Mathematics and Economics, 1, 277-285. doi:10.1016/0167-6687(82)90028-2

12. Derrig, R.A. and Ostaszewski, K. (1997). Managing the tax liability of a property liability insurance company. Journal of Risk and Insurance, 64, 695-711. doi: $10.2307 / 253892$

13. Dubois, D. and Prade, H. (1980). Fuzzy sets and systems: theory and applications. In the serie: Mathematics in Science and Engineering Vol. 144. Academic Press: New York.

14. Dubois, D. and Prade, H. (1993). Fuzzy numbers: an overview. In: Dubois, D., Prade, H. and Yager, R.R. (eds.) Fuzzy Sets for intelligent systems, 113-148. Morgan Kaufmann Publishers: San Mateo (California).

15. Feng, Y., Hu, L. and Shu, H. (2001). The variance and covariance of fuzzy random variables and their application. Fuzzy Sets and Systems, 120, 487-497. doi:10.1016/S0165-0114(99)00060-3

16. Heberle, J. and Thomas, A. (2014) Combining chain-ladder reserving with fuzzy numbers. Insurance: Mathematics and Economics, 55, 96-104. doi:10.1016/j.insmatheco.2014.01.002

17. Huang, T., Zhao, R. and Tang, W. (2009). Risk model with fuzzy random individual claim amount. European Journal of Operational Research, 192(3), 879-890. doi:10.1016/j.ejor.2007.10.035

18. Inuiguchi, M. and Ramık, J. (2000). Possibilistic linear programming: a brief review of fuzzy mathematical programming and a comparison with stochastic programming in portfolio selection problem. Fuzzy Sets and Systems, 111(1), 3-28. doi:10.1016/S01650114(98)00449-7

19. Kaufmann, A. (1986). Fuzzy subsets applications in OR and management. In: Fuzzy Sets Theory and Application, 257-300. Springer: Netherlands.

20. Körner, R. (1997). On the variance of fuzzy random variables. Fuzzy Sets and Systems, 92, 83-93. doi:10.1016/S0165-0114(96)00169-8 
21. Krätschmer, V. (2001). A unified approach to fuzzy random variables. Fuzzy Sets and Systems, 123, 1-9. doi:10.1016/S0165-0114(00)00038-5

22. Kruse, R. and Meyer, K.D. (1987). Statistics with Vague Data, volume 33. Reidel: Dordrecht.

23. Kwakernaak, H. (1978). Fuzzy random variables I: Definitions and Theorems. Information Sciences, 15, 1-29. doi:10.1016/0020-0255(78)90019-1

24. Kwakernaak, H. (1979). Fuzzy random variables II. Algorithms and examples for the discrete case. Information Sciences, 17(3), 253-278. doi:10.1016/00200255(79)90020-3.

25. Lemaire, J. (1990). Fuzzy insurance. Astin Bulletin, 20, 33-55. doi:10.2143/AST.20.1.2005482.

26. Li Calzi, M. (1990). Towards a general setting for the fuzzy mathematics of finance. Fuzzy Sets and Systems, 35, 265-280. doi:10.1016/0165-0114(90)90001-M

27. Li, D. X. and Panjer, H. H. (1994). Immunization measures for life contingencies. In: $4^{\text {th }}$ AFIR Conference, 375-395.

28. López-Diaz, M., and Gil, M. A. (1998). The $\lambda$-average value and the fuzzy expectation of a fuzzy random variable. Fuzzy Sets and Systems, 99(3), 347-352. doi:10.1016/S01650114(97)00031-6.

29. Näther, W. (2000). On random fuzzy variables of second order and their application to linear statistical inference with fuzzy data. Metrika, 51(3), 201-221. doi: $10.1007 / \mathrm{s} 001840000047$

30. Ostaszewski, K. (1993). An investigation into possible applications of fuzzy sets methods in actuarial science, Society of Actuaries: Schaumburg.

31. Pitacco, E. (1986). Simulation in insurance. In: Goovaerts, M., de Vylder, F. and Haezendonck, J. Insurance and risk theory, 59-62. Reidel: Dordretch.

32. Puri, M.L. and Ralescu, D.A. (1986). Fuzzy random variables. Journal of Mathematical Analysis and Applications, 114, 409-422. doi:10.1016/0022-247X(86)90093-4

33. Shapiro, A. (2004). Fuzzy logic in insurance. Insurance: Mathematics and Economics, 35, 399-424. doi:10.1016/j.insmatheco.2004.07.010

34. Shapiro, A. (2009). Fuzzy random variables. Insurance: Mathematics and Economics, 44, 307-314. doi:10.1016/j.insmatheco.2008.05.008

35. Shapiro, A. (2013) Modeling future lifetime as a fuzzy random variable. Insurance: Mathematics and Economics, 53, 864-870. doi:10.1016/j.insmatheco.2013.10.007

36. Viertl, R. and Hareter, D. (2004). Fuzzy information and stochastics. Iranian Journal of Fuzzy Systems, 1, 43-56.

37. Wang, G. and Zhang, Y. (1992). The theory of fuzzy stochastic processes. Fuzzy Sets and Systems, 51(2), 161-178. doi:10.1016/0165-0114(92)90189-B

38. Wilmoth, J.R., Andreev, K., Jdanov, D., Glei, D.A., Boe, C., Bubenheim, M. and Vachon, P. (2007). Methods protocol for the human mortality database. University of California, Berkeley, and Max Planck Institute for Demographic Research, Rostock. URL: http://mortality. org [version 31/05/2007]

39. Wolthuis, H. and Van Hoek, I. (1986). Stochastic models for life contingencies. Insurance: Mathematics and Economics, 5(3), 217-254. doi:10.1016/01676687(86)90034-X 
40. Zadeh, L. (1965). Fuzzy sets. Information and Control, 8, 338-353. doi:10.1016/S00199958(65)90241-X

41. Zmeskal, Z. (2005). Value at risk methodology under soft conditions approach (fuzzystochastic approach). European Journal of Operational Research 161, 337-345. doi:10.1016/j.ejor.2003.08.048 